R. Gangolli and G. Warner

Nagoya Math. J.

Vol. 78 (1980), 1-44

\title{
ZETA FUNCTIONS OF SELBERG'S TYPE FOR SOME NONCOMPACT QUOTIENTS OF SYMMETRIC SPACES OF RANK ONE
}

\author{
RAMESH GANGOLLI* AND GARTH WARNER*
}

\section{§ 0. Introduction}

In a previous paper [5], one of the present authors has worked out a theory of zeta functions of Selberg's type for compact quotients of symmetric spaces of rank one. In the present paper, we consider the analogues of those results when $G / K$ is a noncompact symmetric space of rank one and $\Gamma$ is a discrete subgroup of $G$ such that $G / \Gamma$ is not compact but such that $\operatorname{vol}(G / \Gamma)<\infty$. Thus, $\Gamma$ is a non-uniform lattice. Certain mild restrictions, which are fulfilled in many arithmetic cases, will be put on $\Gamma$, and we shall consider how one can define a zeta function $Z_{\Gamma}$ of Selberg's type attached to the data $(G, K, \Gamma)$.

This will be attempted as in [5], by first defining, via the trace formula, the logarithmic derivative $z_{\Gamma}$ of $Z_{\Gamma}$. The main reason why we get somewhere is the fact that in the case $\operatorname{rank}(G / K)=1$, the parabolic terms in the trace formula can be fully evaluated, for a spherical admissible function $f$, in terms of the spherical Fourier transform $\hat{f}$. The computations necessary for this were performed by the present authors, and were reported in [21].

For technical reasons, presently to be explained, we shall eventually exclude the case $G=S U(2 n, 1)$. Except for this case, our results for $Z_{r}$ are pretty satisfactory. We shall see that $Z_{\Gamma}$ is a meromorphic function. The location and orders of its zeroes and poles will turn out to contain topological and/or spectral information. It will also be seen that $Z_{r}$ enjoys a functional equation which involves not only the Harish-Chandra $c$-function (which determines the Plancherel measure of $G / K$ ) but also the determinant $\Psi(s)$ of the intertwining operator $M(s)$ which occurs in the

Received May 2, 1978.

* Work supported partially by the National Science Foundation. 
theory of Eisenstein series as developed in the last twenty years by Selberg and Langlands.

The restriction $G \neq S U(2 n, 1)$ comes in for a purely technical reason. In studying the analytic continuation of $z_{\Gamma}$, one writes it as a sum $\sum_{i=1}^{7} A_{i}(s)$ and studies $A_{i}(s)$ separately. For our method to work, it is essential that each $A_{i}(s)$ should have the following property: $A_{i}(s)$ has a meromorphic continuation to $C$ with simple poles at which the residues are integral. That this is the case can be proved for all the terms $A_{i}(s)$ except for $A_{6}(s)$. For that particular term, one sees that if $G \neq S U(2 n, 1)$ then $A_{6}(s) \equiv 0$ so the above property is trivial. We were not able to prove the property of meromorphy for $A_{6}(s)$ in general. It seems to us that it is conceivable that $A_{6}(s)$ has the desired property, and a more determined analysis of the term $A_{6}(s)$ will yield the expected result. Thus the lacuna in our theory seems to be due not to any fault of the method, but due to peculiar structural facts about $G=S U(2 n, 1)$, which lead to a complicated expression for $A_{6}(s)$. Such difficulties have arisen in the same case $(G=S U(2 n, 1))$ in other work as well, see e.g. [13], [21], and we are compelled to leave this lacuna in our theory unfilled at this time.

In the course of proving the results about $Z_{\Gamma}$, it is necessary to get some a priori information concerning the asymptotic behavior of the spherical part of the discrete spectrum in $L^{2}(G / \Gamma)$. A little more precisely, suppose that $\left\{U_{j}\right\}_{j \geq 0}$ are the spherical representations of $G$ that occur discretely in $L^{2}(G / \Gamma)$, let $\left\{n_{j}\right\}_{j \geq 0}$ be their multiplicities and let $\omega_{j}$ be the eigenvalue of the Casimir element in the representation $U_{j}$. For any $r>0$, we define the Weyl function $N$ by $N(r)=\sum_{\left\{j:\left|\omega_{j}\right| \leq r\right\}} n_{j}$. Some information about the asymptotic behavior of $N(r)$ as $r \rightarrow \infty$ is needed for our study of $Z_{\Gamma}$. If $G / \Gamma$ is compact, it is well known that $N(r) \sim C_{G} r^{n / 2}$ where $n=\operatorname{dim}(G / K)$, and $C_{G}$ is a constant depending only on $G$. In our case, where $G / \Gamma$ is not compact, there is a difficulty in proving such a precise result. The difficulty is due to our ignorance of the asymptotic behavior of $\Psi^{\prime} \mid \Psi$ along the imaginary axis, $\Psi$ being the function referred to above. If it could be shown that $\mid \Psi^{\prime}($ ir $) / \Psi($ ir $) \mid \leq$ Const. $|r|^{d}$ for $r$ real, and some $d \leq n$, then it is possible to get the asymptotic result $N(r) \sim$ $C_{G} r^{n / 2}$. In all the known cases where $\Psi$ can be computed, this property of $\Psi$ can be verified, but it follows from fairly subtle estimates for the size of the Riemann zeta function and certain Dirichlet $L$-functions. In the absence of detailed information about $\Psi$ in the general case, one 
cannot get the precise estimate $N(r) \sim C_{G} r^{n / 2}$. Nevertheless, we are able to prove that $N(r)$ is tempered, i.e. for some integer $k$ we have $N(r) \sim C r^{k}$ as $r \rightarrow \infty$. This fact turns out to be sufficient for our purpose, which is to study $Z_{\Gamma}$.

Finally, the analytic information about $Z_{\Gamma}$ (or rather about its logarithmic derivative $z_{\Gamma}$ ) enables us to prove a result about the "length spectrum' of the noncompact manifold $K \backslash G / \Gamma$. This manifold has the homotopy type of a compact manifold, and the length spectrum is the set of lengths of minimizing geodesics in the free homotopy classes of closed paths on $K \backslash G / \Gamma$. The asymptotic behavior of the distribution function of these lengths can be determined. In the compact case, analogous results have been proved for $G=S L(2, R)$ in [10], and for general $G$ in [1], [4].

In $\S 1$, we deal with preliminaries. $\S 2$ is devoted to a discussion of the Weyl function $N(r)$. In $\S 3$, we define the logarithmic derivative $z_{\Gamma}$ of $Z_{\Gamma}$ and study its analytic continuation. $\S 4$ is devoted to studying $Z_{\Gamma}$ and its properties. The functional equation is derived there. $\S 5$ contains the result on the length spectrum. An appendix contains a technical result used in $\S 2$.

We would like to acknowledge our indebtedness to the referee for a very careful reading of the manuscript which brought to light a factor in the functional equation in $\S 4$ which we had inadvertently overlooked, and also corrected several mistakes of sign in $\S 3$ which made the original version confusing.

\section{§1. Preliminaries and the Trace Formula}

Let $G$ be a connected noncompact simple Lie group with finite center, $K$ a maximal compact subgroup. Denote by $\mathfrak{g}, \mathfrak{f}$ their respective Lie algebras, and $\mathfrak{g}=\mathfrak{f}+\mathfrak{p}$ the Cartan decomposition with Cartan involution $\theta$. We denote by $\langle$,$\rangle the Cartan-Killing form and put |X|_{\theta}^{2}=\langle X, X\rangle_{\theta}$. Then $|\cdot|_{\theta}$ is a norm on $g$. We endow $g$ with the Euclidean structure corresponding to $\langle\cdot, \cdot\rangle_{\theta}$.

Let $\mathfrak{a}_{\mathfrak{p}}$ be a maximal abelian subspace of $\mathfrak{p}$. Extend $\mathfrak{a}_{\mathfrak{p}}$ to a $\theta$-stable maximal abelian subalgebra, say $\mathfrak{a}$, of $\mathfrak{g}$, and put $\mathfrak{a}_{\mathfrak{f}}=\mathfrak{a} \cap \mathfrak{f}$. We denote by $\mathfrak{g}^{C}, \mathfrak{f}^{C}, \mathfrak{a}^{C}$ etc. the complexifications of $\mathfrak{g}, \mathfrak{f}, \mathfrak{a}$ etc. Then $\mathfrak{a}^{C}$ is a Cartan subalgebra of $g^{c}$. Let $\Phi=\Phi\left(\mathfrak{g}^{c}, \mathfrak{a}^{c}\right)$ be the set of roots of $\left(\mathfrak{g}^{c}, \mathfrak{a}^{c}\right)$, and introducing, as usual, compatible orders on the duals of $\mathfrak{a}_{\mathfrak{p}}$ and $\mathfrak{a}_{\mathfrak{p}}+i \mathfrak{a}_{\mathfrak{t}}$, 
let $\Phi^{+}$be the set of positive roots in $\Phi$. Put $P_{+}=\left\{\alpha \in \Phi^{+} \mid \alpha \neq \equiv\right.$ on $\left.a_{p}\right\}$ and $P_{-}=\Phi^{+}-P_{+}$. Put $\rho=\frac{1}{2} \sum_{\alpha \in P_{+}} \alpha$.

Let $\mathfrak{g}=\mathfrak{l}+\mathfrak{a}_{\mathfrak{p}}+\mathfrak{n}, G=K A_{\mathfrak{p}} N$ be the Iwasawa decompositions corresponding to these choices $\left(A_{\mathfrak{p}} \stackrel{\text { def }}{=} \exp \mathfrak{a}_{\mathfrak{p}}, N \stackrel{\text { def }}{=} \exp \mathfrak{n}\right)$. Denote by $M$ the centralizer of $A_{p}$ in $K$, and let $P=M A_{p} N$ be the minimal parabolic subgroup determined by these choices. For $x \in G$ define $H(x) \in \mathfrak{a}_{\mathfrak{p}}$ by $x=$ $k \exp H(x) n(k \in K, n \in N)$. We denote by $W$ the Weyl group of $\left(G, A_{\mathfrak{q}}\right)$.

We assume throughout this paper that $\operatorname{rank}(G / K)=1$.

Let $\Sigma$ be the set of restrictions to $\mathfrak{a}_{\mathfrak{p}}$ of the elements of $P_{+}$. Then we can find an element $\beta \in \Sigma$ such that $2 \beta$ is the only other possible element of $\Sigma$. Let $p, q$ equal, respectively, the number of elements of $P_{+}$which restrict to $\beta$ and $2 \beta$. We fix an element $H_{0}$ of $\mathfrak{a}_{\mathfrak{p}}$ by the condition $\beta\left(H_{0}\right)$ $=1$. Then one knows that $\left\langle H_{0}, H_{0}\right\rangle_{\theta}=2 p+8 q$ and $\rho\left(H_{0}\right)=(p+2 q) / 2$. We shall often denote by $c_{0}$ the number $\left|H_{0}\right|_{\theta}=(2 p+8 q)^{1 / 2}$ and by $\rho_{0}$ the number $\rho\left(H_{0}\right)$.

Let $\Lambda$ be the dual space of $\mathfrak{a}_{\mathfrak{p}}, \Lambda^{c}=\Lambda+i \Lambda$ its complexification. For, $\lambda \in \Lambda^{c}$, we write $\lambda=\operatorname{Re}(\lambda)+i \operatorname{Im}(\lambda)$, where $\operatorname{Re}(\lambda), \operatorname{Im}(\lambda) \in \Lambda$. Since $\operatorname{dim} a_{p}$ $=1$, we have $\Lambda \cong R, \Lambda^{C} \cong C$, and we fix the following identification of $\Lambda^{\boldsymbol{C}}$ with $\boldsymbol{C}$ for future use: Namely $s \in \boldsymbol{C}$ shall correspond to $s \beta \in \Lambda^{\boldsymbol{C}}$.

We denote by $C^{\infty}(K \backslash G / K)$ (resp. $C_{c}^{\infty}(K \backslash G / K)$ ) the spaces of smooth spherical (i.e. $K$-biinvariant) functions on $G$ (resp. smooth compactly supported spherical functions on $G)$. The spaces $L^{1}(K \backslash G / K), L^{2}(K \backslash G / K)$ etc. are defined similarly. The functions $\Xi(x)$ and $\sigma(x)$ are defined as in [19] and one defines, as usual, Harish-Chandra's Schwartz space $\mathscr{C}(G)$ (cf. [19]). The $L^{p}$ analogue $\mathscr{C}^{p}(G)$ is defined, as in [19], as follows: Let $D_{1}, D_{2}$ be respectively left and right invariant differential operators on $G, r$ an integer, and for $f \in C^{\infty}(G)$, put

$$
\tau_{D_{1}, D_{2}, r}^{p}(f)=\sup _{x \in G} \Xi(x)^{-2 / p}(1+\sigma(x))^{r}\left|f\left(D_{1} i x ; D_{2}\right)\right|
$$

where we use the notation of [19]. The space $\mathscr{C}^{p}(G)$ consists of those $f \in C^{\infty}(G)$ for which $\tau_{D_{1}, D_{2}, r}^{p}(f)<\infty$ for each $D_{1}, D_{2}, r . \mathscr{C}^{p}(G)$ is a Fréchet space with $\tau_{D_{1}, D_{2}, r}^{p}$ as semi-norms. When $p=2$, we have $\mathscr{C}^{p}(G)=\mathscr{C}(G) \subset$ $L^{2}(G)$. In general, $\mathscr{C}^{p}(G) \subset L^{p}(G), p>0$, and $\mathscr{C}^{p}(G) \subseteq \mathscr{C}^{p^{\prime}}(G)$ if $p \leq p^{\prime}$. The subspace of $K$-biinvariant functions in $\mathscr{C}^{p}(G)$ will be denoted by $\mathscr{C}^{p}(K \backslash G / K)$.

We fix the normalized Haar measure $d k$ on $K$, and the Haar measure $d n$ on $N$ normalized by the condition 


$$
\int_{N} \exp -2 \rho(H(\bar{n})) d n=1
$$

where $\bar{n}=\theta\left(n^{-1}\right)$. Next fix the Haar measure $d h$ on $A_{p}$ as follows: For $h \in A_{\mathfrak{p}}$ put $u(h)=\beta(\log h)$. Then $u=u(h)$ is a coordinate function on $A_{\mathfrak{p}}$, and $h \rightarrow u(h)$ is an isomorphism of $A_{\mathfrak{p}}$ with $\boldsymbol{R}$. Let $d u$ be the Lebesgue measure on $R$, and $d h$ the Haar measure corresponding to it via this isomorphism. Observe that for $h \in A_{p}$, we have $h=\exp u H_{0}$ with $u=u(h)$ $=\beta(\log h)$.

We now normalize the Haar measure $d x$ on $G$ by $d x=\exp 2 \rho(\log h)$ $d k d h d n$, if $x=k h n$ (cf. [19]). These normalizations remain fixed throughout this paper.

For $\nu \in \Lambda^{c}$, we denote by $\phi_{\nu}$ the elementary (or zonal) spherical function corresponding to $\nu$, defined by

$$
\phi_{\nu}(x)=\int_{K} \exp (i \nu-\rho)(H(x k)) d k .
$$

One has then the spherical Fourier transform $\hat{f}$ and the Abel-HarishChandra transform $F_{f}$ defined by

$$
\begin{gathered}
\hat{f}(\nu)=\int_{G} f(x) \phi_{\nu}(x) d x, \quad \nu \in \Lambda^{c} \\
F_{f}(h)=\exp \rho(\log h) \int_{N} f(h n) d n, \quad h \in A_{\mathfrak{v}}
\end{gathered}
$$

for any $f \in \mathscr{C}(K \backslash G / K)$, and the relation

$$
\hat{f}(\nu)=F_{f}^{*}(\nu)=\int_{A_{\mathfrak{p}}} F_{f}(h) \exp i \nu(\log h) d h .
$$

$F_{f}^{*}$ is the Euclidean Fourier transform on the vector group $A_{p}$.

Fix the Haar measure $d \nu$ on $A$ by requiring that the measures $d h$ and $d \nu$ are dual in the sense of Fourier analysis, namely that we shall have

$$
g(1)=\int_{A} g^{*}(\nu) d \nu \quad \text { for } g \in C_{c}^{\infty}\left(A_{\downarrow}\right) .
$$

Then we have the inversion formula for the transform $f \rightarrow \hat{f}$, given by

$$
f(1)=[W]^{-1} \int_{\Lambda} \hat{f}(\nu) c(\nu)^{-1} c(-\nu)^{-1} d \nu
$$

for $f \in \mathscr{C}(K \backslash G \mid K)$, where [W] is the order of $W$ and $c(\cdot)$ is the well-known $c$-function of Harish-Chandra [8]. It is known that $c(\nu)=\overline{c(-\nu)}$ for $\nu \in \Lambda$, 
and $c(\nu)^{-1} c(-\nu)^{-1} d \nu=|c(\nu)|^{-2} d \nu$ is the Plancherel measure for $L^{2}(K \backslash G / K)$.

As in [5], we write $r(\nu)=\nu\left(H_{0}\right)$ for $\nu \in \Lambda$. Then $r=r(\nu)$ is a parameter on $\Lambda$, and it sets up an isomorphism of $\Lambda$ with $R$. Let $d r$ be the usual Lebesgue measure on $R$. The measure $d \nu$ on $\Lambda$ fixed above corresponds to the measure $d r / 2 \pi$ under this parametrization. For $f \in \mathscr{C}^{p}(K \backslash G / K)$, $p \leq 2$, the formulas (1.4)-(1.8) can be written in terms of the parameters $r, u$ and it is best to set them out, since we shall use them in that form. For $f \in \mathscr{C}^{p}(K \backslash G / K), p \leq 2$, define $F$ on $R$ by

$$
F(u)=F_{f}(h) \quad \text { where } u=u(h)
$$

and let $F^{*}$ be the ordinary Fourier transform of $F$,

$$
F^{*}(r)=\int_{-\infty}^{\infty} F(u) \exp (i r u) d u
$$

Then

$$
F^{*}(r)=F_{f}^{*}(\nu)=\hat{f}(\nu), \quad r=r(\nu)
$$

and

$$
\begin{aligned}
F(u) & =F_{f}(h) \\
& =(1 / 2 \pi) \int_{-\infty}^{\infty} F^{*}(r) \exp (-i r u) d r \quad(u=u(h)) .
\end{aligned}
$$

The function $c$ is given by

$$
c(\nu)^{-1}=\frac{\Gamma((p+q) / 2) \Gamma(i r+p / 2) \Gamma(i r / 2+(p+2 q) / 4)}{\Gamma(p+q) \Gamma(i r) \Gamma(i r / 2+p / 4)},
$$

where $r=r(\nu)$ and $\Gamma(\cdot)$ is the classical gamma function. The inversion formula (1.8) reads

$$
\begin{aligned}
f(1) & =[W]^{-1} / 2 \pi \int_{-\infty}^{\infty} F^{*}(r) c(r)^{-1} c(-r)^{-1} d r \\
& =(1 / 4 \pi) \int_{-\infty}^{\infty} F^{*}(r) c(r)^{-1} c(-r)^{-1} d r
\end{aligned}
$$

since $[W]=2$.

Now let $\Gamma$ be a discrete subgroup of $G$ such that $G / \Gamma$ has finite volume, but is not compact. We normalize the $G$-invariant measure $d \dot{x}$ on $G / \Gamma$ so that the formula

$$
\int_{G} f(x) d x=\int_{G / \Gamma}\left\{\sum_{\gamma \in \Gamma} f(x \gamma)\right\} d \dot{x}
$$


holds for $f \in C_{c}(G)$.

Let $d$ be the number of equivalence classes of $\Gamma$-cuspidal minimal parabolic subgroups of $G$, the equivalence relation being conjugacy by an element of $\Gamma$. We fix parabolic subgroups $P^{1}(=P), P^{2}, \cdots, P^{d}$ to represent these classes. One knows that we can find elements $k_{i} \in K$, such that $P^{i}=k_{i} P k_{i}^{-1}, 1 \leq i \leq d$; moreover, $P^{i}=M^{i} A_{p}^{i} N^{i}$ is the Langlands decomposition of $P^{i}$, with $M^{i}=k_{i} M k_{i}^{-1}$ etc. (of course $k_{1}=1$ ).

By transport of structure, every concept defined relative to $P$ will give rise to an analogous concept relative to $P^{i}$. We shall denote it by adding a superscript $i$. Thus for example $\beta^{i}$ will stand for the root $\beta$ transported to $\mathfrak{a}_{\mathfrak{p} .}^{i}$ Similarly $H^{i}(x), \rho^{i}, \nu^{i}$ have obvious interpretations.

We assume that $\Gamma$ satisfies the following conditions.

(1.16) Let $Z(\Gamma)$ be the center of $\Gamma$. Then $\Gamma \cap P^{i}=Z(\Gamma)\left(\Gamma \cap N^{i}\right)(1 \leq i \leq d)$.

(1.17) $\Gamma$ has no elements of finite order, other than those in $Z(\Gamma)$.

It is known that $Z(\Gamma)$ is contained in $K$ and is therefore finite.

We now write down the Selberg Trace Formula in the form that we wish to use. The technique of Langlands for obtaining this formula was explained in detail in [21]. In $\S 9$ of that paper, some computations done by the present authors were also reported. We shall need those results. Some familiarity with [21] may be useful. We stress that the normalizations of the various Haar measures we use here differ from those used in [21]. We hope that no confusion will be caused by this.

Let $U$ be the left regular representation of $G$ on $L^{2}(G / \Gamma)$. As we know, $L^{2}(G / \Gamma)$ then breaks up as the sum of three mutually orthogonal closed $U$-stable subspaces which we call $L_{\text {cus }}^{2}(G / \Gamma), L_{\mathrm{Eis}}^{2}(G / \Gamma)$ and $L_{\mathrm{res}}^{2}(G / \Gamma)$. They are respectively, the closures of the subspaces spanned by the cusp forms, the wave packets formed with Eisenstein series, and the square-integrable residues of the Eisenstein series (cf. [21]). The restriction of $U$ to $L_{\text {cus }}^{2}(G / \Gamma) \oplus L_{\text {res }}^{2}(G / \Gamma)$ is a discrete direct sum of irreducible unitary representations of $G$, with finite multiplicities, while the restriction of $U$ to $L_{\mathrm{Eis}}^{2}(G / \Gamma)$ is a direct integral of unitary principal series representations. For this reason we write $L_{d}^{2}(G / \Gamma)=L_{\text {cus }}^{2}(G / \Gamma) \oplus L_{\text {res }}^{2}(G / \Gamma)$ and $L_{c}^{2}(G / \Gamma)=$ $L_{\mathrm{Eis}}^{2}(G / \Gamma)$, and call these respectively, the discrete and continuous pieces of $L^{2}(G / \Gamma)$.

Thanks to the work of Selberg and Langlands, the space $L_{c}^{2}(G / \Gamma)$ is 
well understood. We shall be concerned with the subspace of $L_{c}^{2}(G / \Gamma)$ which consists of those $f$ such that $f(k x)=f(x)(k \in K, x \in G)$. We denote it by $L_{c}^{2}(K \backslash G / \Gamma)$. It is the closure of the subspace spanned by wave packets formed with Eisenstein series of type $1(1 \in \hat{K}$ being the class of the trivial representation of $K)$. Since we shall have to be involved in some aspects of the theory of these series, we describe briefly what we need.

Let $P^{i}=M^{i} A_{p}^{i} N^{i}$ be one of our $\Gamma$-cuspidal parabolic subgroups, and let $\nu^{i} \in \Lambda^{i, c}$. The Eisenstein series $E\left(P^{i}, \nu^{i}, x\right)$ is defined in $\left\{\nu^{i} \in \Lambda^{i, c} \mid \operatorname{Re}\left(\nu^{i}\right)\right.$ $\left.>\rho^{i}\right\}$ by

$$
E\left(P^{i}, \nu^{i}, x\right)=\sum_{\gamma \in \Gamma / \Gamma \cap P^{i}} \exp \left(\nu^{i}+\rho^{i}\right)\left(H^{i}(x \gamma)\right) .
$$

This series converges absolutely, uniformly on compact subsets of $\left\{\nu^{i} \in \Lambda^{i, C} \mid \operatorname{Re}\left(\nu^{i}\right)>\rho^{i}\right\} \times G$. For fixed $x \in G$ it is a holomorphic function of $\nu^{i}$, it is a left $K$-invariant, right $\Gamma$-invariant eigenfunction of every element of the center of the enveloping algebra of $G$.

The constant term of $E\left(P^{i}, \nu^{i}, x\right)$ along $P^{j}$ is by definition the function $E_{P^{j}}\left(P^{i}, \nu^{i}, x\right)$ where

$$
E_{P^{j}}\left(P^{i}, \nu^{i}, x\right)=\left(\operatorname{vol}\left(N^{j} / \Gamma \cap N^{j}\right)\right)^{-1} \int_{N^{j} / \Gamma \cap N^{j}} E\left(P^{i}, \nu^{i}, x n^{j}\right) d \dot{n}^{j} .
$$

Here $d \dot{n}^{j}$ is the measure on $N^{j} / \Gamma \cap N^{j}$ induced by $d n^{j}$ (which has been fixed), when we endow $\Gamma \cap N^{j}$ with counting measure.

One has

$$
E_{P^{j}}\left(P^{i}, \nu^{i}, x\right)=\sum_{w \in W_{i j}} M_{i j}\left(w, \nu^{i}\right) \exp \left(w \nu^{i}+\rho^{j}\right)\left(H^{j}(x)\right)
$$

where $W_{i j}$ is the set of bijections from $A^{i} \rightarrow A^{j}$ induced by inner automorphisms of $G$, and $M_{i j}\left(w, \nu^{i}\right)$ are certain complex numbers. For fixed $w \in W_{i j}$, the function $\nu^{i} \rightarrow M_{i j}\left(w, \nu^{i}\right)$ is holomorphic in $\left\{\nu^{i} \in \Lambda^{i, c} \mid \operatorname{Re}\left(\nu^{i}\right)>\rho^{i}\right\}$.

Now let $w$ be the non-trivial element of $W$ and define the $d \times d$ matrix-valued function $M(\nu)$ on $\left\{\nu \in \Lambda^{c} \mid \operatorname{Re}(\nu)>\rho\right\}$ by

$$
M_{i j}(\nu)=M_{i j}\left(k_{j} w k_{i}^{-1}, k_{i} \nu\right) .
$$

The central result of the theory of Eisenstein series, applied to our case, is as follows: Let $E(\nu, x)$ be the column vector with entries $E\left(P^{i}, \nu^{i}\right.$, $x),\left(\nu^{i}=k_{i} \nu\right)$-then the functions $E(\nu, x), M(\nu)$ have an analytic continuation to all $\nu \in \Lambda^{c}$ and are meromorphic when so continued. The poles of $E(\nu, x)$ 
are independent of $x$, and belong to the set of poles of $M(\nu)$, and we have the functional equation

$$
E(\nu, x)=M(\nu) E(-\nu, x), \quad \nu \in \Lambda^{c} .
$$

The function $M$ has no poles in $\operatorname{Re}(\nu)>\rho$, and its poles in $\operatorname{Re}(\nu)>0$ are known to be finite in number; they are all simple, and lie on the line segment $\{\nu \in \Lambda \mid 0<\nu \leq \rho\}$. The poles in $\operatorname{Re}(\nu)<0$ are not known. $M$ satisfies the functional equations

$$
\begin{gathered}
M(\nu) M(-\nu)=\text { Identity } \\
M^{*}(\nu)=M(\bar{\nu})
\end{gathered}
$$

where $M^{*}$ is the adjoint of $M$ with respect to the Hermitian structure on $\Lambda^{C}$ given by $\langle\cdot, \cdot\rangle_{\theta}$. In particular, when $\nu \in i \Lambda$, we see that $M(\nu)$ is unitary, and hence holomorphic on $i \Lambda$. We shall sometimes refer to $M$ as the intertwining function of $\Gamma$. These results are specializations of those in [12]. $\Psi(\nu)$ will stand for $\operatorname{det} M(\nu)$.

In the sequel, we shall often use for $\nu \in \Lambda^{c}$, the parameter $s=s(\nu)=$ $\nu\left(H_{0}\right)$ to parametrize $\Lambda^{c}$. By means of this parametrization, the function $M$ becomes a meromorphic function on $C$, and we have

$$
\begin{gathered}
M(s) M(-s)=\text { Identity } \\
M^{*}(s)=M(\bar{s}) .
\end{gathered}
$$

Similarly $\Psi$ will be viewed as a function on $\boldsymbol{C}$. We have

$$
\begin{gathered}
\Psi(s) \Psi(-s)=1 \\
\Psi(s)=\Psi(\bar{s}) .
\end{gathered}
$$

The following lemma will be used below.

LEMMA 1.1. $\Psi^{\prime}(s) \Psi(s)^{-1}$ is a meromorphic function having simple poles and integer residues, and we have $\Psi^{\prime}(s) \Psi(s)^{-1}=$ trace $\left(M^{\prime}(s) M(s)^{-1}\right), s \in C$.

Proof. Since $\Psi(s)=\operatorname{det} M(s)$, and $M$ is meromorphic, so is $\Psi$. Hence $\Psi^{\prime} \mid \Psi$ has simple poles with integer residues. Next, to prove the equality $\Psi^{\prime} \mid \Psi=$ trace $\left(M^{\prime} M^{-1}\right)$, we need only prove it for $s$ in an open set. Fix $s_{0} \in C$ such that $M\left(s_{0}\right)$ is non-singular and put $R(s)=M\left(s_{0}\right)^{-1} M(s)$. Then $M(s)=M\left(s_{0}\right) R(s)$. If $s$ is near to $s_{0}$, say in a neighborhood $U$, we have $R(s)$ near to the identity matrix, so we can write $R(s)=\exp A(s)$ where 
$A$ is holomorphic in $U$. It is easily seen that if $\tilde{\Psi}(s)=\operatorname{det} R(s)$, then $\tilde{\Psi}(s)=\exp (\operatorname{trace} A(s))$, so $\tilde{\Psi}^{\prime} \mid \tilde{\Psi}=\operatorname{trace}\left(A^{\prime}(s)\right)=\operatorname{trace}\left(R^{\prime}(s) R(s)^{-1}\right)$. But it is easy to check that $\tilde{\Psi}^{\prime}\left|\tilde{\Psi}=\Psi^{\prime}\right| \Psi$, and that

$$
\operatorname{trace}\left(R^{\prime}(s) R(s)^{-1}\right)=\operatorname{trace}\left(M^{\prime}(s) M(s)^{-1}\right) .
$$

Hence our assertion follows.

We are indebted to David Ragozin for suggesting this proof, which is simpler than the one we had originally put in.

The nature of the function $\Psi^{\prime} \mid \Psi$ will be quite important to us. Not much is known about $\Psi$ in general. However, when $\operatorname{rank}(G / K)=1$, it can be shown that $\Psi$ is the quotient of two entire functions, say $P$ and $Q$, both of finite order. This result, due to Selberg for $G=S L(2, R)$, can be generalized to any $G$ such that $\operatorname{rank}(G / K)=1$, as is seen by an examination of the work in [2], [20]. The generalization depends, as is seen from these works, on the fact that $\operatorname{dim} \Lambda=1$, and proceeds as in the classical case of $G=S L(2, R)$. We shall use this result freely below.

As we have noted above, the restriction of $U$ to $L_{d}^{2}(G / \Gamma)$ is discretely decomposable with finite multiplicities. Let $U_{1}, U_{2}, \cdots$ be a complete list of mutually inequivalent irreducible unitary representations of class one which occur in $L_{d}^{2}(G / \Gamma)$, and let $n_{j}$ be the multiplicity of $U_{j}$. Each $n_{j}$ is finite. Moreover, as observed in [21, § 8] for example, only a finite number of the $U_{j}$ can occur in $L_{\text {res }}^{2}(G / \Gamma)$. Since $U_{1}$ of class one, it corresponds to a unique elementary positive definite spherical function, say, $\phi_{\nu j}$, with $\nu_{j} \in \Lambda^{C} . \quad \nu_{j}$ is defined uniquely up to an action of $W$. Since $\phi_{\nu j}$ is positive definite, one knows that $\nu_{j}$ is either purely real on $a_{p}$ or purely imaginary on $\mathfrak{a}_{\mathfrak{p}}$. We pick $\nu_{j}$ in such a way that if $\nu_{j}$ is purely real, we will have $\nu_{j}\left(H_{0}\right) \geq 0$, while, if it is purely imaginary, we will have $\operatorname{Im}\left(\nu_{j}\left(H_{0}\right)\right) \geq 0$. This fixes the various $\nu_{j}$ unambiguously (cf. [5]).

We shall write $r_{j}^{+}=\nu_{j}\left(H_{0}\right), r_{j}^{-}=-\nu_{j}\left(H_{0}\right)$ and put $s_{j}^{+}=\rho_{0}+i r_{j}^{+}, s_{j}^{-}=$ $\rho_{0}+i r_{j}^{-}, j \geq 0$. Then $\left(r_{j}^{+}\right)^{2}$ is real and lies in $\left[-\rho_{0}^{2}, \infty\right)$. Also either (i) $\operatorname{Re}\left(s_{j}^{+}\right)=\rho_{0}$ or (ii) $\operatorname{Im}\left(s_{j}^{+}\right)=0$ and $s_{j}^{+}$lies in $\left[0, \rho_{0}\right)$. Note also that $s_{0}^{+}=0$.

Let $\gamma \in \Gamma$ be a semisimple element which is not elliptic, i.e. which is not conjugate to any element of $K$, and let $G_{\gamma}$ be its centralizer in $G$. Then $\gamma$ is conjugate to some element of $A$, say $h(\gamma)$. Let $h(\gamma)=h_{t}(\gamma) h_{p}(\gamma)$, with $h_{t}(\gamma) \in A_{\mathfrak{t}}, h_{\mathfrak{p}}(\gamma) \in A_{\mathfrak{p}}$. We can always choose $h(\gamma)$ so that $\beta\left(\log h_{\mathfrak{p}}(\gamma)\right)$ $>0$. We shall assume that we have picked and fixed $h(\gamma)$ with this property. As observed in [4], the centralizer of $h(\gamma)$ in $G$ is equal to 
$M_{h(r)} A_{p}$, where $M_{h(r)}$ is the centralizer of $h(\gamma)$ in $K$. We normalize the Haar measure on $M_{h(\gamma)} A_{\mathfrak{p}}$ so that the compact group $M_{h(r)}$ carries normalized Haar measure, and $A_{\mathfrak{p}}$ carries the Haar measure $d h$ fixed above. Since $G_{r}$ is conjugate to $M_{h(r)} A_{p}$, this gives us a unique measure on $G_{r}$, which is in fact independent of the choices made in defining it. Having fixed this measure on $G_{r}$, we call it $d x_{r}$, and endow $\Gamma_{r}\left(=G_{r} \cap \Gamma\right)$ with counting measure. These normalizations force us into unique choices of invariant measures $d \dot{x}_{\gamma}$ on $G_{r} / \Gamma_{r}$, and $d x_{r}^{*}$ on $G / G_{r}$ in the usual way, so that we have $d x=d x_{r}^{*} d x_{r}$ etc. Finally let $C_{\Gamma}$ stand for a complete set of representatives for $\Gamma$-conjugacy classes of semisimple elements in $\Gamma$.

The following result was proved in $[21, \S 8]$, in a more general form.

Theorem 1.2. Let $f \in \mathscr{C}^{p}(K \backslash G / K)$, with $p<1$. Then the restriction of $U(f)$ to $L_{d}^{2}(G / \Gamma)$ is a trace class operator, whose trace is given by either side of the following trace formula.

$$
\begin{aligned}
\sum_{j=1}^{\infty} n_{j} \hat{f}\left(\nu_{j}\right)= & {[Z(\Gamma)] \operatorname{vol}(G / \Gamma) f(1) } \\
& +\sum_{r \in G=\Gamma^{-Z(\Gamma)}} \operatorname{vol}\left(G_{\gamma} / \Gamma_{\gamma}\right) \int_{G / G_{\gamma}} f\left(x \gamma x^{-1}\right) d x_{\gamma}^{*} \\
& +\lim _{s \rightarrow 0} \frac{d}{d s}\left(s \Psi_{f}(s)\right) \\
& +[W]^{-1} \int_{\Lambda} \hat{f}(\nu) \operatorname{trace}\left(M^{\prime}(i \nu) M(i \nu)^{-1}\right) d \nu \\
& -\frac{1}{4} \hat{f}(0) \operatorname{trace}(M(0)) .
\end{aligned}
$$

Here, $s$ is a complex variable and $\Psi_{f}(s)$ is a certain family of distributions, depending analytically on $s$, discussed fully in $\S 6,8$ of [21]. The above theorem is just an application of Theorem 8.4 of [21], bearing in mind that our $f$ is spherical.

For fixed $f \in \mathscr{C}^{p}(K \backslash G / K), p<1$, the function $s \rightarrow \mathrm{\Psi}_{f}(s)$ was shown to be meromorphic in a vertical strip containing $s=0 ; \Psi_{f}(s)$ has a simple pole at $s=0$ and so $\lim _{s \rightarrow 0}(d / d s)\left(s \mathrm{U}_{f}(s)\right)$ is just the constant term in the solved; Laurent expansion of $\mathrm{U}_{f}$ about $s=0$.

The problem of computing $\lim _{s \rightarrow 0}(d / d s)\left(s \Psi_{f}(s)\right)$ is not yet completely cf. [13] for a partial solution. The present authors, in $\S 9$ of [21], did however compute it for $f \in \mathscr{C}^{p}(K \backslash G / K)$. We quote it here.

Theorem 1.3. For $f \in \mathscr{C}^{p}(K \backslash G / K)$ with $p<1$, we have 


$$
\lim _{s \rightarrow 0}(d / d s)\left(s \mathrm{Ч}_{f}(s)\right)=C_{\beta}(\Gamma) T_{\beta}(f)+C_{\beta}^{\prime}(\Gamma) T_{\beta}^{\prime}(f)+C_{2 \beta}(\Gamma) T_{2 \beta}(f) .
$$

Here $C_{\beta}(\Gamma), C_{\beta}^{\prime}(\Gamma)$ and $C_{2 \beta}(\Gamma)$ are certain constants depending on ( $G$, $K, \Gamma)$, and $C_{2 \beta}(\Gamma)=0$ if $N$ is abelian. Moreover $T_{\beta}(f), T_{\beta}^{\prime}(f)$ and $T_{2 \beta}^{\prime}(f)$ are certain distributions fully discussed in $\S 9$ of [21]. (They were called $T_{\lambda}(\alpha: 0), T_{\lambda}^{\prime}(\alpha: 0)$ and $T_{2 \lambda}(\alpha: 0)$ there). More precisely we have, allowing for the differences in normalizations of the measures, from Theorems 9.3 and 9.4 of [21], that

$$
\begin{gathered}
C_{\beta}(\Gamma) T_{\beta}(f)=\kappa_{1} \int_{\Lambda} \hat{f}(\nu) d \nu \\
C_{\beta}^{\prime}(\Gamma) T_{\beta}^{\prime}(f)=\kappa_{2} \int_{\Lambda} \hat{f}(\nu) d \nu+d / 4 \hat{f}(0) \\
-d \int_{\Lambda} \hat{f}(\nu) \Gamma^{\prime}\left(1+i \nu\left(H_{0}\right)\right) / \Gamma\left(1+i \nu\left(H_{0}\right)\right) d \nu
\end{gathered}
$$

and

$$
C_{2 \beta}(\Gamma) T_{2 \beta}(f)=\kappa_{3} \int_{\Lambda} \hat{f}(\nu) J(\nu) d v
$$

where $\kappa_{1}, \kappa_{2}, \kappa_{3}$ are constants depending on $(G, K, \Gamma)$, whose exact value will not concern us, and $J$ is an entire function on $\Lambda^{c}$, whose nature depends on $(G, K)$, and which we need to explicate a little bit more here because we shall need these facts later in $\S 3$. The formulas for $J$ were written out in $\S 9$ of [21]. We quickly recapitulate them. In doing so, we shall generically denote by $C$ any constant depending only on $(G, K)$, and not necessarily the same in different locations. We have (cf. [21, Theorem 9.4])

$$
\begin{aligned}
T_{2 \beta}(f)=C I_{k, k}\left(F_{f}\right) & \text { if } p=4 k, k \in Z \\
& =C J_{k, k}\left(F_{f}\right) \quad \text { if } p=4 k-2, k \in Z
\end{aligned}
$$

where $I_{k, k}, J_{k, k}$ are certain distributions that were explicitly computed in $[21, \S 9]$, and were expressed in terms of $\hat{f}$. More specifically, we have

$$
I_{k, k}\left(F_{f}\right)=\sum_{\ell} a_{\ell}(k) I_{\ell, 0}\left(F_{f}\right)+\sum_{\ell} b_{\ell}(k) I_{\ell, 1}\left(F_{f}\right)
$$

where the sums are finite, $a_{\ell}(k), b_{\ell}(k)$ are integers, and $I_{\ell, 0}, I_{\ell, 1}$ are certain distributions. In fact

$$
I_{\ell, 0}\left(F_{f}\right)=\int_{\Lambda} \hat{f}(\nu) p_{\ell}(\nu) d \nu
$$




$$
I_{\ell, 1}\left(F_{f}\right)=\int_{\Lambda} \hat{f}(\nu) q_{\ell}(\nu) d \nu
$$

where $p_{\ell}$ and $q_{\ell}$ are even polynomials in $\nu$ (i.e. $p_{\ell}(\nu)=p_{\ell}(-\nu)$ and $q_{\ell}(\nu)=$ $\left.q_{\ell}(-\nu)\right)$. As to $J_{k, k}$, we have

$$
J_{k, k}\left(F_{f}\right)=\sum_{\ell} c_{\ell}(k) J_{\ell, 0}\left(F_{f}\right)+\sum_{\ell} d_{\ell}(k) J_{\ell, 1}\left(F_{f}\right)
$$

where the sums are finite, $c_{\ell}(k), d_{\ell}(k)$ are integers, and $J_{\ell, 0}, J_{\ell, 1}$ are certain distributions. In fact

$$
\begin{aligned}
J_{\ell, 0}\left(F_{f}\right) & =\int_{0}^{\infty}\left(D^{2 \ell} F\right)(u) d u \\
& =\frac{1}{2} \int_{\Lambda} \hat{f}(\nu) G_{\ell}(\nu) d \nu
\end{aligned}
$$

where $D$ is the operator $(\sinh u)^{-1} d / d u, F(u)=F_{f}(h)$ if $u=u(h)$, and $G_{\ell}(\nu)$ is given by

$$
G_{\ell}(\nu)=\frac{1}{\pi} \int_{0}^{\infty} D^{2 \ell}\left(\cos \left(\nu\left(H_{0}\right) u\right)\right) d u
$$

similarly,

$$
\begin{aligned}
J_{\ell, 1}\left(F_{f}\right) & =-\int_{0}^{\infty}\left(D^{2 \ell-1} F\right)(u) \cosh u d u \\
& =\frac{1}{2} \int_{\Lambda} \hat{f}(\nu) H_{\ell}(\nu) d \nu
\end{aligned}
$$

where

$$
H_{\ell}(\nu)=\frac{1}{4} \int_{-\infty}^{\infty} F_{\ell}(\nu, \mu) \frac{\mu \sinh \pi \mu}{\cosh \pi \mu-1} d \mu
$$

and

$$
F_{\ell}(\nu, \mu)=\frac{2}{\pi} \int_{0}^{\infty} D^{2 \ell-2}\left(\cos \left(\nu\left(H_{0}\right) u\right)\right) \cos \mu u d u .
$$

Summarizing all this information, we see that the function $J(\nu)$ referred to above is entire. In fact it is an even polynomial whenever the integer $p$ is $\equiv 0(\bmod 4)$. When $p$ is of the form $4 k-2$, the formulas above make it plain that $J(\nu)$ is a finite linear combination of the functions $G_{\ell}(\nu)$ and $H_{\ell}(\nu)$ described above. These facts will be important to us in $\S 3$.

(1.29) now reads as follows. 


$$
\begin{aligned}
\sum_{j \geq 0} n_{j} \hat{f}\left(\nu_{j}\right)= & {[Z(\Gamma)] \operatorname{vol}(G / \Gamma) f(1) } \\
& +\sum_{r \in C_{\Gamma}^{-}-Z(\Gamma)} \operatorname{vol}\left(G_{r} / \Gamma_{r}\right) \int_{G / G_{r}} f\left(x \gamma x^{-1}\right) d x_{r}^{*} \\
& +\frac{1}{4}(d-\operatorname{trace}(M(0))) \hat{f}(0) \\
& -d \int_{\Lambda} \hat{f}(\nu) \Gamma^{\prime}\left(1+i \nu\left(H_{0}\right)\right) / \Gamma\left(1+i \nu\left(H_{0}\right)\right) d \nu \\
& +\kappa_{4} \int_{\Lambda} \hat{f}(\nu) d \nu \\
& +\kappa_{3} \int_{\Lambda} \hat{f}(\nu) J(\nu) d \nu \\
& +\frac{1}{4 \pi} \int_{\Lambda} \hat{f}(\nu) \operatorname{trace}\left(M^{\prime}(i \nu)^{-1} M(i \nu)^{-1}\right) d \nu .
\end{aligned}
$$

For a semisimple element $\gamma$, we have, as in [4],

$$
\operatorname{vol}\left(G_{\gamma} / \Gamma_{\gamma}\right) \int_{G / G_{\gamma}} f\left(x \gamma x^{-1}\right) d x_{r}^{*}=\beta\left(\log h_{p}(\gamma)\right) j(\gamma)^{-1} C(h(\gamma)) F_{f}\left(h_{p}(\gamma)\right) .
$$

Here, $j(\gamma)$ is the positive integer such that $\gamma=\delta^{j(r)}$ with $\delta$ primitive in $\Gamma$, cf. [4], and $C(\cdot)$ is the positive function on $A_{p}$ defined by

$$
C(h)=\varepsilon_{R}^{A}(h) \xi_{p}\left(h_{\rho}(\gamma)\right)^{-1} \prod_{\alpha \in P_{+}}\left(1-\xi_{\alpha}(h(\gamma))^{-1}\right)^{-1} .
$$

Expressing $F_{f}$ in terms of its Fourier transform $F_{f}^{*}$ (which equals $\hat{f}$ ), we can write

$$
\begin{aligned}
\sum_{j \geq 0} n_{j} F^{*}\left(r_{j}^{+}\right)= & {[Z(\Gamma)] \operatorname{vol}(G / \Gamma) \frac{1}{4 \pi} \int_{-\infty}^{\infty} F^{*}(r) c(r)^{-1} c(-r)^{-1} d r } \\
& +\sum_{r \in C_{\Gamma^{-}} Z(\Gamma)} u\left(h_{p}(\gamma)\right) j(\gamma)^{-1} C(h(\gamma)) \frac{1}{2 \pi} \int_{-\infty}^{\infty} F^{*}(r) \exp -\left(i r u\left(h_{p}(\gamma)\right) d r\right. \\
& +\frac{1}{4} F^{*}(0)(d-\operatorname{trace}(M(0))) \\
& -\frac{d}{2 \pi} \int_{-\infty}^{\infty} F^{*}(r) \Gamma^{\prime}(1+i r) / \Gamma(i+i r) d r \\
& +\kappa_{5} \int_{-\infty}^{\infty} F^{*}(r) d r+\kappa_{6} \int_{-\infty}^{\infty} F^{*}(r) J(r) d r \\
& +\frac{1}{4 \pi} \int_{-\infty}^{\infty} F^{*}(r) \operatorname{trace}\left(M^{\prime}(i r) M(i r)^{-1}\right) d r
\end{aligned}
$$

where the kappas are constants depending on $(G, K, \Gamma)$, and $J$ is the function discussed above (of course we now write $J(r)=J(\nu)$ if $r=r(\nu)=$ 
$\left.\nu\left(H_{0}\right)\right)$. Remember that $u(h)=\beta(\log h)$. In the sequel we shall often write $u_{\gamma}$ for $u\left(h_{p}(\gamma)\right)=\beta\left(\log h_{p}(\gamma)\right)$. Then $u_{\gamma}>0$, by our choice of $h(\gamma)$ and $h_{p}(\gamma)$.

It will be useful to have a condition on $F^{*}$ which will imply that the corresponding $f$ lies in $\mathscr{C}^{p}(K \backslash G / K)$ for some $p<1$. This can be done as in [17]; for example, if $F^{*}$ satisfies (i) $F^{*}(z)=F^{*}(-z)$, (ii) for some $\varepsilon>0$, $F^{*}$ is holomorphic in the strip $\left\{z \in C|| \operatorname{Im}(z) \mid \leq \rho_{0}+\varepsilon\right\}$ and (iii) $F^{*}$ is rapidly decreasing as a function of $\operatorname{Re}(z)$ on the boundaries of that strip, then one can show that there exists $p<1$ and an $f \in \mathscr{C}^{p}(K \backslash G / K)$ such that $\hat{f}(\nu)=$ $F^{*}(r)(r=r(\nu))$. Such an $f$ is admissible for the trace formula.

Finally, we have the following proposition [6].

Proposition 1.4. There exists an integer $m$ such that $\sum_{j \geq 0} n_{j}\left(1+\left(r_{j}^{+}\right)^{2}\right.$ $\left.+\rho_{0}^{2}\right)^{-m}<\infty$.

In particular, the numbers $r_{j}^{+}$have no finite point of accumulation in $C$.

\section{§2. An estimate for the spectrum}

We will need an estimate for the Weyl distribution function $N(r)$ defined for any $r>0$ by

$$
N(r)=\sum_{\left\{j:\left(r_{j}^{+}\right)^{2} \leq r\right\}} n_{j} .
$$

In the case where $G / \Gamma$ is compact, one has the estimate $N(r) \sim$ $C_{G} \operatorname{vol}(G / \Gamma) r^{n / 2}$ as $r \rightarrow \infty$ where $n=\operatorname{dim}(G / K)$ [3]. It would be nice to have a similar estimate in the present case. Unfortunately, we are not able to get such a precise estimate due to our ignorance of the detailed behavior of the function trace $\left(M^{\prime}(i r) M(i r)^{-1}\right)$. However, we are able to get a less precise estimate for $N(r)$, which will suffice for the applications that we have in mind. As we will point out more fully below, the precise asymptotic estimate can be proved if we are willing to assume some moderation in the growth of the function $r \rightarrow \operatorname{trace}\left(M^{\prime}(i r) M(i r)^{-1}\right)$.

To study the behavior of $N(r)$, we use the fundamental solution $h_{t}$ of the heat equation on $G / K$ as in [5, p. 22]. Thus $h_{t}$ is the fundamental solution of the heat equation $\Omega u=c_{0}^{2}(\partial u / \partial t)$ where $c_{0}^{2}=\left|H_{0}\right|_{\theta}^{2}=2 p+8 q$. As pointed out in $\left[5\right.$, p. 22], we have $\hat{h}_{t}(\nu)=\exp -\left(r(\nu)^{2}+\rho_{0}^{2}\right) t$ for any $t>0$. Thus, if we write $H_{t}^{*}(r)=\hat{h}_{t}(\nu), r=r(\nu)$, we have $H_{t}^{*}(r)=\exp -\left(r^{2}+\rho_{0}^{2}\right) t$. It is clear that $h_{t}$ is admissible, and the trace formula applied to $h_{t}$ yields the following: 


$$
\begin{aligned}
\sum_{j \geq 0} n_{j} \exp -\left(\left(r_{j}^{+}\right)^{2}+\rho_{0}^{2}\right) t \\
=[Z(\Gamma)] \operatorname{vol}(G / \Gamma) \frac{1}{4 \pi} \int_{-\infty}^{\infty} \exp -\left(r^{2}+\rho_{0}^{2}\right) t \cdot c(r)^{-1} c(-r)^{-1} d r \\
\quad+(4 \pi t)^{-1 / 2} \sum_{r \in C} \sum_{\Gamma^{-} Z(\Gamma)}\left|u_{r}\right| j(\gamma)^{-1} C(h(\gamma)) \exp -\left(\rho_{0}^{2} t+u_{r}^{2} / 4 t\right) \\
\quad+\frac{1}{4 \pi} \int_{-\infty}^{\infty} \exp -\left(r^{2}+\rho_{0}^{2}\right) t \cdot \Psi^{\prime}(i r) \Psi(i r)^{-1} d r \\
\quad+\frac{1}{4} \exp -\rho_{0}^{2} t \cdot(d-\operatorname{trace}(M(0))) \\
\quad-\frac{d}{2 \pi} \int_{-\infty}^{\infty} \exp -\left(r^{2}+\rho_{0}^{2}\right) t \cdot \Gamma^{\prime}(1+i r) / \Gamma(1+i r) d r \\
\quad+\kappa_{5} \int_{-\infty}^{\infty} \exp -\left(r^{2}+\rho_{0}^{2}\right) t d r \\
\quad+\kappa_{6} \int_{-\infty}^{\infty} \exp -\left(r^{2}+\rho_{0}^{2}\right) t \cdot J(r) d r .
\end{aligned}
$$

Now let $L(t)$ be the Laplace transform of the measure $d N(r)$. Thus, by definition,

$$
L(t)=\int_{0}^{\infty} e^{-r t} d N(r)=\sum_{j \geq 0} n_{j} \exp -\left(r_{j}^{+}\right)^{2} t .
$$

One finds from (2.2) that we have

$$
\begin{aligned}
L(t)=[ & Z(\Gamma)] \operatorname{vol}(G / \Gamma) \frac{1}{4 \pi} \int_{-\infty}^{\infty} \exp -r^{2} t \cdot c(r)^{-1} c(-r)^{-1} d r \\
& +(4 \pi t)^{-1 / 2} \sum_{\gamma}\left|u_{r}\right| j(\gamma)^{-1} C(h(\gamma)) \exp -u_{r}^{2} / 4 t \\
& +\frac{1}{4 \pi} \int_{-\infty}^{\infty} \exp -r^{2} t \cdot \Psi^{\prime}(i r) \Psi(i r)^{-1} d r+\frac{1}{4}(d-\operatorname{trace}(M(0))) \\
& -\frac{d}{2 \pi} \int_{-\infty}^{\infty} \exp -r^{2} t \cdot \Gamma^{\prime}(1+i r) / \Gamma(1+i r) d r \\
& +\kappa_{5} \int_{-\infty}^{\infty} \exp -r^{2} t d r+\kappa_{6} \int_{-\infty}^{\infty} \exp -r^{2} t \cdot J(r) d r .
\end{aligned}
$$

We will label the various terms on the right $J_{1}(t), J_{2}(t), \cdots, J_{7}(t)$. We want to study $\lim _{t \rightarrow 0} t^{n / 2} L(t)$ for $n=\operatorname{dim}(G / K)$. Ideally, we would like $\lim _{t \rightarrow 0} t^{n / 2} L(t)=C_{G} \operatorname{vol}(G / \Gamma)$. In fact, as in [3], we find that $\lim _{t \rightarrow 0} t^{n / 2} J_{1}(t)$ $=C_{G} \operatorname{vol}(G / \Gamma)$ where $C_{G}$ is a constant depending only on $G$, so the issue is reduced to a study of $\lim _{t \rightarrow 0} t^{n / 2} J_{i}(t), 2 \leq i \leq 7$. The term $J_{2}(t)$ can be dealt with as in [3]. We have $\lim _{t \rightarrow 0} t^{n / 2} J_{2}(t)=0 . J_{3}$ is the troublesome term, which we discuss below. It is clear that $\lim _{t \rightarrow 0} t^{n / 2} J_{4}(t)=\lim _{t \rightarrow 0} t^{n / 2} J_{6}(t)=0$. As for $J_{5}(t)$, observe that we have the classical estimate (cf. [11, p. 317]) 


$$
\left|\Gamma^{\prime}(1+i r)\right| \Gamma(1+i r) \mid \leq \text { Const. } \log |r|,|r| \geq 2 .
$$

It follows that $\lim _{t \rightarrow 0} t^{n / 2} J_{5}(t)=0$ for every $n \geq 1$. Similarly, using the expression for $J(r)$ in $\S 1$, we conclude that $|J(r)| \leq$ Const. $|r|^{k}$, where $k \leq$ $\operatorname{dim}(G / K)$. This of course needs a careful reference to [21, §9], and in particular, needs a calculation of the integer $k_{\lambda}$ mentioned there. Anyhow, the upshot is that

$$
\left|J_{7}(t)\right| \leq \text { Const. } \int_{0}^{\infty} \exp -r^{2} t \cdot r^{k} d r \leq \text { Const. } \int_{0}^{\infty} \exp -u t \cdot u^{k / 2-1 / 2} d u .
$$

It follows from an application of the Tauberian theorem for the Laplace transform that $\lim _{t \rightarrow 0} t^{n / 2} J_{7}(t)=0$, because $k \leq n=\operatorname{dim}(G / K)$. Thus we have dealt with all the terms $J_{i}(t)$ except the term $J_{3}(t)$.

Clearly, if one could show that

$$
\left|\Psi^{\prime}(i r) \Psi(i r)^{-1}\right| \leq \text { Const. }|r|^{k}
$$

with $k \leq \operatorname{dim}(G / K)$, the above argument can be repeated to yield $\lim _{t \rightarrow 0} t^{n / 2} J_{3}(t)=0$. Now, in all the cases where $\Psi$ can be explicitly computed, one can show that (2.6) is true, and, in fact, a much better estimate of the form

$$
\left|\Psi^{\prime}(i r) \Psi(i r)^{-1}\right| \leq \text { Const. }(\log |r|)^{k}
$$

holds. This is because in all the known cases, the function $\Psi(s)$ can be expressed in terms of $\Gamma$-functions, the Riemann zeta function, and Dirichlet $L$-functions (cf. [9], [15], [16]) and then one can invoke classical estimates for the logarithmic derivatives of these functions. It would be interesting if a non-computational method could be found which would yield the estimate (2.7) or, what is adequate for our purposes, the estimate (2.6), with $k \leq \operatorname{dim}(G / K)$.

While we cannot prove (2.6) in general, we can show that there exists an integer $k$ such that $N(r) \leq C r^{k}$. We shall now describe how such an estimate can be made. If it could be shown that (2.6) holds with an integer $\leq n$, we would of course have $N(r) \sim C_{G} r^{n / 2}$.

In $\S 8$ of [21], it was proved that all the terms in the trace formula (as written there) are continuous linear functionals on a certain function space, called $\mathscr{C}_{s}^{1}(G)$ there, $\varepsilon$ being any positive real number. This conclusion is the content of Theorem 8.4 of [21]. $\mathscr{C}_{6}^{1}(G)$ is the space $\mathscr{C}^{p}(G)$, with $p=2 /(2+\varepsilon)$; observe that $p<1$. We can therefore conclude that if 
$f \in \mathscr{C}^{p}(K \backslash G / K)$, then the linear functional $T(f)$ defined by

$$
T: f \rightarrow \int_{\Lambda} \hat{f}(\nu) \operatorname{trace}\left(M^{\prime}(i \nu) M(i \nu)^{-1}\right) d \nu
$$

is continuous on the Fréchet space $\mathscr{C}^{p}(K \backslash G / K)$, for every $p<1$. If we write the integral in terms of the parameter $r=r(\nu)$, we conclude that the assignment

$$
T: f \rightarrow \int_{-\infty}^{\infty} F^{*}(r) \Psi^{\prime}(i r) \Psi(i r)^{-1} d r
$$

where $F^{*}(r)=f(\nu), r=r(\nu)$, is continuous on $\mathscr{C}^{p}(K \backslash G / K)$, if $p<1$.

Now fix $p<1$-then the Fréchet space $\mathscr{C}^{p}(K \backslash G / K)$ is isomorphic (via the transform $f \rightarrow \hat{f}$ ) to the Fréchet space $\mathscr{F}_{p}$ (cf. [17]) consisting of all even functions $\hat{f}$ holomorphic on the strip $S_{p}=\left\{\nu \in \Lambda^{c}|| \operatorname{Im}\left(\nu\left(H_{0}\right)\right) \mid \leq \rho_{0}(2 / p-1)\right\}$ and for which every seminorm $\tau(\hat{f})$ defined below is finite. The seminorms $\tau$ are defined by

$$
\tau(\hat{f})=\sup _{\lambda \in S_{p}}|P(\lambda)(D \hat{f})(\lambda)|
$$

where $P$ is a polynomial and $D$ is a differential operator with constant coefficients.

The topology given by the seminorms $\tau$ defined above is equivalently also given by the seminorms $\tau_{k, \ell},(k, \ell \geq 0$, integral), defined by

$$
\tau_{k, \ell}\left(F^{*}\right)=\sup _{r \in S_{p}}\left|\left(1+r^{2}\right)^{k} \frac{d^{\ell}}{d r^{\ell}} F^{*}(r)\right|
$$

Thus, the continuity of the distribution $f \rightarrow T(f)$ defined in (2.9) amounts to the following assertion.

LEMMA 2.1. Let $f \in \mathscr{C}^{p}(K \backslash G / K)$, and put $F^{*}(r)=\hat{f}(\nu)$ for $r=r(\nu)$. Then there exist integers $k, \ell$ such that we have

$$
\left|\int_{-\infty}^{\infty} F^{*}(r) \Psi^{\prime}(i r) \Psi(i r)^{-1} d r\right| \leq \tau_{k, \ell}\left(F^{*}\right) .
$$

Now considering the function $h_{t}$ as above, we note that $h_{t}$ is in $\mathscr{C}^{p}(K \backslash G / K)$, so that (2.12) holds for $H_{t}^{*}$ in place of $F^{*}$. Recalling that $H_{t}^{*}(r)=\exp -t\left(r^{2}+\rho_{0}^{2}\right)$, it follows that for suitable $k, \ell$ we have, after cancelling a factor of $\exp -t \rho_{0}^{2}$, 


$$
\left|\int_{-\infty}^{\infty} \exp -r^{2} t \cdot \Psi^{\prime}(i r) \Psi(i r)^{-1} d r\right| \leq \sup _{r \in S_{s}}\left|\left(1+r^{2}\right)^{k} \frac{d^{\ell}}{d r^{\ell}}\left(\exp -r^{2} t\right)\right|
$$

In other words

$$
\left|J_{2}(t)\right| \leq \sup _{r \in S_{\varepsilon}}\left|\left(1+r^{2}\right)^{k} \frac{d^{\ell}}{d r^{\ell}}\left(\exp -r^{2} t\right)\right|
$$

for suitable $k, \ell$.

It is easy to see that the right side can be estimated by Const. $t^{-N}$ for $t \leq 1$, and $N$ some non-negative integer. See the appendix. It follows that for any integer $k>N$, we shall have

$$
\lim _{t \rightarrow 0} t^{k} J_{3}(t)=0
$$

We note that the above discussion shows that there exists an integer $k$ such that $\lim _{t \rightarrow 0} t^{k} L(t)=0$. By applying the Tauberian theorem for the Laplace transform, we can deduce

Proposition 2.2. The Weyl function $N(r)$ is tempered. More precisely, there exists a positive integer $k$ and a constant $C>0$ such that $N(r) \leq C r^{k}$ for all $r>0$.

It should be noted that if we could obtain a more precise idea of which seminorm $\tau$ can be used in (2.13) we would be able to get a precise estimate for $k$.

\section{§3. Definition of $z_{\Gamma}$ and $Z_{r}$}

As in [5], we want to define the logarithmic derivative of $Z_{F}$, and study its analytic continuation.

Let $\varepsilon_{0}>0$ be fixed, and let $g$ be a real-valued function in $C^{\infty}(R)$ such that (i) $g$ is even (ii) $g$ vanishes in a neighbourhood of 0 , (iii) $g$ is constant, equal to $c$ say for $|x| \geq \varepsilon_{0}$, and (iv) $0 \leq g \leq c$. The values of $c, \varepsilon_{0}$ will be chosen later.

For $s \in C$, put

$$
g(s, u)=g(|u|) \exp \left(\left(\rho_{0}-s\right)|u|\right), \quad u \in \boldsymbol{R} .
$$

Then for fixed $s, g$ is smooth, even and $g(s, u)=c \exp \left(\left(\rho_{0}-s\right)|u|\right)$ if $|u| \geq \varepsilon_{0}$.

Now suppose that $\operatorname{Re}(s)>2 \rho_{0}$. Then by the remarks at the end of $\S 1$, we can find $p(=p(s))<1$ and an $f_{s} \in \mathscr{C}^{p}(K \backslash G / K)$ such that $F_{f_{s}}(h)=$ 
$g(s, u(h))$. We shall put $F_{s}(h)=F_{f_{s}}(h)=g(s, u(h))$. Since $f_{s}$ is admissible, we get (cf. [5]),

$$
\begin{aligned}
\sum_{j \geq 0} n_{j} \hat{f}_{s}\left(\nu_{j}\right)= & {[Z(\Gamma)] \operatorname{vol}(G / \Gamma) f_{s}(1) } \\
& +\sum_{r \in C_{\Gamma^{-Z}(\Gamma)}} u_{r} j(\gamma)^{-1} C(h(\gamma)) g\left(s, u_{r}\right) \\
& +\frac{1}{4 \pi} \int_{-\infty}^{\infty} F_{s}^{*}(r) \operatorname{trace}\left(M^{\prime}(i r) M(i r)^{-1}\right) d r \\
& +\frac{1}{4} F_{s}^{*}(0)(d-\operatorname{trace}(M(0))) \\
& -\frac{d}{2 \pi} \int_{-\infty}^{\infty} F_{s}^{*}(r) \cdot \Gamma^{\prime}(1+i r) / \Gamma(1+i r) d r+\kappa_{5} \int_{-\infty}^{\infty} F_{s}^{*}(r) d r \\
& +\kappa_{6} \int_{-\infty}^{\infty} F_{s}^{*}(r) J(r) d r .
\end{aligned}
$$

The numbers $\left|u_{r}\right|, \gamma \in C_{\Gamma}-Z(\Gamma)$, are bounded away from zero (this is an easy deduction from the convergence of the series on the right side of (1.47)). Fix $\varepsilon_{0}>0$ so small that it is smaller than all of the numbers $|u|_{r}$. Then $g\left(s, u_{r}\right)=c \exp \left(\left(\rho_{0}-s\right)\left|u_{r}\right|\right)$. Thus, we get

Proposition 3.1. The series

$$
z_{\Gamma}(s, g)=g\left(\varepsilon_{0}\right) \sum_{\gamma \in C^{-}-Z(\Gamma)}\left|u_{\gamma}\right| j(\gamma)^{-1} C(h(\gamma)) \exp \left(\left(\rho_{0}-s\right)\left|u_{r}\right|\right)
$$

converges absolutely for each $s$ in $\operatorname{Re}(s)>2 \rho_{0}$. The convergence is uniform for $s$ in any half plane of the form $\operatorname{Re}(s) \geq 2 \rho_{0}+\varepsilon, \varepsilon>0$.

Observe that, because of (3.2) we have

$$
\begin{aligned}
z_{\Gamma}(s, g)= & \sum_{j \geq 0} n_{j} \hat{f}_{s}\left(\nu_{j}\right)-f_{s}(1)[Z(\Gamma)] \operatorname{vol}(G / \Gamma)-\frac{1}{4} F_{s}^{*}(0)(d-\operatorname{trace}(M(0))) \\
& +\frac{d}{2 \pi} \int_{-\infty}^{\infty} F_{s}^{*}(r) \Gamma^{\prime}(1+i r) / \Gamma(1+i r) d r-\kappa_{5} \int_{-\infty}^{\infty} F_{s}^{*}(r) d r \\
& -\kappa_{6} \int_{-\infty}^{\infty} F_{s}^{*}(r) J(r) d r-\frac{1}{4 \pi} \int_{-\infty}^{\infty} F_{s}^{*}(r) \operatorname{trace}\left(M^{\prime}(i r) M(i r)^{-1}\right) d r .
\end{aligned}
$$

There are seven terms on the right side. We shall call them $A_{1}(s)$, $A_{2}(s), \cdots, A_{7}(s)$. We shall now study the analytic continuation of each of these terms.

For any complex $r$, put

$$
H(r)=\int_{0}^{\infty} g^{\prime}(u) \exp (i r u) d u .
$$


As in [5], we have the lemmas:

LEMMA 3.2. $H$ is an entire function of $r$. Moreover, for each integer $n \geq 1$, we can find $C_{n}>0$ such that

$$
\begin{aligned}
|H(r)| & \leq C_{n}|r|^{-n} \quad \text { if } \operatorname{Im}(r) \geq 0 \\
& \leq C_{n}|r|^{-n} \exp \left(\varepsilon_{0}|\operatorname{Im}(r)|\right) \quad \text { if } \operatorname{Im}(r)<0 .
\end{aligned}
$$

Lemma 3.3. Let $f_{s}$ be as above, with $\operatorname{Re}(s)>2 \rho_{0}$. Then all for $\nu \in \Lambda^{c}$ for which $f_{s}(\nu)$ makes sense, we have

$$
\hat{f}_{s}(\nu)=\frac{H\left(i s-i \rho_{0}+\nu\left(H_{0}\right)\right)}{s-\rho_{0}-i \nu\left(H_{0}\right)}+\frac{H\left(i s-i \rho_{0}-\nu\left(H_{0}\right)\right)}{s-\rho_{0}+i \nu\left(H_{0}\right)} .
$$

The estimate for $H$ and the formula for $\hat{f}_{s}$ are at the basis of the investigation of the $A_{i}(s)$.

Proposition 3.4. The function $A_{1}(s)=\sum_{j \geq 0} n_{j} \hat{f}_{s}\left(\nu_{j}\right)$, defined for $\operatorname{Re}(s)$ $>2 \rho_{0}$, has a meromorphic continuation to the whole complex plane. Its poles are simple, and are located at the points $s_{j}^{+}, s_{j}^{-}, j \geq 0$, where $s_{j}^{+}=$ $\rho_{0}+i \nu_{j}\left(H_{0}\right), s_{j}^{-}=\rho_{0}-i \nu_{j}\left(H_{0}\right)$. The residues at both $s_{j}^{+}$and $s_{j}^{-}$equal $H(0) n_{j}$, if $s_{j}^{+} \neq s_{j}^{-}$. (It is understood that the poles at $s_{0}^{+}$and $s_{0}^{-}$are present only if $\left.n_{0}>0\right)$. Finally, if $s_{j}^{+}=s_{j}^{-}$for some $j$, the residue of $A_{1}(s)$ at $s_{j}^{+}$is $2 n_{j} H(0)$.

Proof. The proof is exactly the same as in [5]. We have

$$
A_{1}(s)=\sum_{j \geq 0} n_{j}\left\{\frac{H\left(i\left(s-s_{j}^{+}\right)\right)}{s-s_{j}^{+}}+\frac{H\left(i\left(s-s_{j}^{-}\right)\right)}{s-s_{j}^{-}}\right\}
$$

in $\operatorname{Re}(s)>2 \rho_{0}$. Each term on the right is meromorphic in $s$, and thanks to Lemma 3.2 and Proposition 1.4, the series converges absolutely, uniformly on compacta disjoint from $\left\{s_{j}^{ \pm}\right\}$and thus defines a meromorphic function in $C$. This proves the proposition.

Proposition 3.5. The term $A_{2}(s)=-f_{s}(1)[Z(\Gamma)]$ vol $(G / \Gamma)$ has a meromorphic continuation to the whole complex plane. In fact we have

$$
A_{2}(s)=-i \operatorname{vol}(G / \Gamma)[Z(\Gamma)] \sum_{k \geq 0} \frac{H\left(i s-i \rho_{0}+r_{k}\right)}{s-\rho_{0}-i r_{k}} \cdot d_{k}
$$

where $\left\{r_{k} \mid k \geq 0\right\}$ are the poles in the upper half-plane of the function $r \rightarrow$ $c(r)^{-1} c(-r)^{-1}$ and $d_{k}$ is the residue of that function at $r_{k}$. The series on the right converges absolutely, uniformly on compact subsets of $C$ disjoint from $\left\{\rho_{0}+i r_{k} \mid k \geq 0\right\}$, and defines the meromorphic continuation of the left side. 
Thus, the function $A_{2}(s)$ has simple poles at $\rho_{0}+i r_{k}, k \geq 0$, with residue $-i[Z(\Gamma)] \operatorname{vol}(G / \Gamma) H(0) d_{k}$ at the pole $\rho_{0}+i r_{k}$.

Proof. The proof differs in no essential way from the one given in [5], hence can be omitted.

It is to be noted that in the event that the function $r \rightarrow c(r)^{-1} c(-r)^{-1}$ has no poles in the upper half-plane (in which case it is actually a polynomial), the term $A_{2}(s)$ is zero. This sort of phenomenon will reappear below in the study of $A_{6}(s)$ and will be very useful to us.

Proposition 3.6. We have

$$
A_{3}(s)=-\frac{1}{2}(d-\operatorname{trace}(M(0))) H\left(i\left(s-\rho_{0}\right)\right) /\left(s-\rho_{0}\right)
$$

for $\operatorname{Re}(s)>2 \rho_{0}$. The right side defines a meromorphic continuation of $A_{3}(s)$ with a simple pole at $s=\rho_{0}$ with residue $-\frac{1}{2} H(0)(d-\operatorname{trace}(M(0)))$.

Proof. We have $F_{s}^{*}(0)=\hat{f}_{s}(0)$. Now use (3.7).

Proposition 3.7. We have

$$
\begin{aligned}
A_{4}(s) & =\frac{d}{2 \pi} \int_{-\infty}^{\infty} F_{s}^{*}(r) \Gamma^{\prime}(1+i r) / \Gamma(1+i r) d r \\
& =-\sum_{k \geq 1} d \frac{H\left(i\left(s-\rho_{0}+k\right)\right)}{s-\rho_{0}+k}
\end{aligned}
$$

for $\operatorname{Re}(s)>2 \rho_{0}$. The series on the right converges absolutely, uniformly on compact subsets of $C$ disjoint from $\left\{\rho_{0}-k \mid k \geq 1\right\}$, and defines a meromorphic continuation of the left side to all of $C$. The poles of $A_{4}(s)$, thus continued, are all simple, and are at the points $\rho_{0}-k, k \geq 1$. The residue at each pole is $-d H(0)$.

Proof. We have

$$
\begin{aligned}
A_{4}(s) & =\frac{d}{2 \pi} \int_{-\infty}^{\infty} F_{s}^{*}(r) \Gamma^{\prime}(1+i r) / \Gamma(1+i r) d r \\
& =\frac{d}{2 \pi} \int_{-\infty}^{\infty}\left(\frac{H\left(i s-i \rho_{0}+r\right)}{s-\rho_{0}-i r}+\frac{H\left(i s-i \rho_{0}-r\right)}{s-\rho_{0}+i r}\right) \frac{\Gamma^{\prime}(1+i r)}{\Gamma(1+i r)} d r .
\end{aligned}
$$

In the integral involving the first term in the parentheses, we shift the integration into the complex plane, to the line $\operatorname{Im}(r)=T$, and let $T \rightarrow \infty$. Using standard estimates on $\Gamma^{\prime} / \Gamma$, and the estimate on $H$ given by Lemma 3.2 , we see that we pick up contributions from the residues of the function $r \rightarrow \Gamma^{\prime}(1+i r) / \Gamma(1+i r)$ in the upper half-plane. The poles of this function 
are at $r=i k, k \geq 1$, and the residue at each pole is equal to $i$. It follows that the first term contributes the series on the right side of (3.11). -Now consider the second term. The integral involving it can be written as

$$
\int_{-\infty}^{\infty} \frac{H\left(i s-i \rho_{0}+r\right)}{s-\rho_{0}-i r} \cdot \frac{\Gamma^{\prime}(1-i r)}{\Gamma(1-i r)} d r .
$$

Once again we shift the integration to $\operatorname{Im}(r)=T$, and use Lemma 3.2 to justify this. But this time $r \rightarrow \Gamma^{\prime}(1-i r) / \Gamma(1-i r)$ has no poles in the upper half plane, and this implies that the integral is zero. Thus (3.11) is verified, and we argue as in the case of $A_{1}(s)$ to prove our proposition.

Proposition 3.8. We have

$$
A_{5}(s) \equiv 0 .
$$

Proof. In fact

$$
\begin{aligned}
A_{5}(s) & =-\kappa_{5} \int_{-\infty}^{\infty} F_{s}^{*}(r) d r \\
& =-\kappa_{5} F_{s}(0) \\
& =-\kappa_{5} g(s, 0) \\
& =0
\end{aligned}
$$

from the definition of $g$.

Proposition 3.9. The term $A_{6}(s)$ is identically zero, provided that $G \neq S U(2 n, 1)$.

Proof. We must show that

$$
\int_{-\infty}^{\infty} F_{s}^{*}(r) J(r) d r=0 \quad \text { for } \operatorname{Re}(s)>2 \rho_{0},
$$

where $J(r)$ is described in $\S 1$. But we have, using the fact that $J(r)$ is even,

$$
\begin{aligned}
\int_{-\infty}^{\infty} F_{s}^{*}(r) J(r) d r \\
\quad=\int_{-\infty}^{\infty}\left(\frac{H\left(i s-i \rho_{0}+r\right)}{s-\rho_{0}-i r}+\frac{H\left(i s-i \rho_{0}-r\right)}{s-\rho_{0}+i r}\right) J(r) d r \\
\quad=2 \int_{-\infty}^{\infty} \frac{H\left(i s-i \rho_{0}+r\right)}{s-\rho_{0}-i r} \cdot J(r) d r .
\end{aligned}
$$

Because we have excluded the case $G=S U(2 n, 1)$, the integer $p$ is $\equiv 0$ 
( $\bmod 4)$, so that $J(r)$ is a polynomial. Let $T>0$, and use Cauchy's theorem on the contour consisting of the semicircle of radius $T$ centered at 0 . We see that

$$
\int_{-T}^{T} \frac{H\left(i s-i \rho_{0}+r\right)}{s-\rho_{0}-i r} J(r) d r
$$

equals the integral of the same integrand along the curved upper semicircle, say $\Omega_{T}$, traversed positively. Now, using Lemma 3.2, we see that the latter integral is $0\left(T^{-n}\right)$ for every integer $n$, since $J(r)$ is a polynomial, and the length of $\Omega_{T}$ is $\pi T$. Letting $T \rightarrow \infty$, we see that (3.14) is indeed zero as claimed.

We now need to discuss the term

$$
A_{7}(s)=-\frac{1}{4 \pi} \int_{-\infty}^{\infty} F_{s}^{*}(r) \operatorname{trace}\left(M^{\prime}(i r) M(i r)^{-1}\right) d r .
$$

Recall that as mentioned in $\S 1$, the function $\Psi$ is in our case a ratio of two entire functions say $P, Q$, both of finite order. Thus

$$
\Psi(s)=P(s) / Q(s) .
$$

We may assume (if necessary, by modifying $P$ and $Q$ by appropriate Weierstrass products) that $P$ and $Q$ have no zeroes in common. Let $\left\{p_{k}\right\}_{k \geq 1}$ be the zeroes of $P,\left\{q_{k}\right\}_{k \geq 1}$ those of $Q$, and let $\left\{a_{k}\right\}_{k \geq 1}\left\{b_{k}\right\}_{k \geq 1}$ be their multiplicities - then the zeroes of $\Psi$ are precisely the $\left\{p_{k}\right\}_{k \geq 1}$ and the poles of $\Psi$ are precisely the $\left\{q_{k}\right\}_{k \geq 1}$. Since $\Psi$ is holomorphic in $\operatorname{Re}(s)>\rho_{0}$, we have $\operatorname{Re}\left(q_{k}\right) \leq \rho_{0}$ for all $k$. Moreover, since $\Psi(s) \Psi(-s)=1$, as a consequence of (1.23), we see that there can be no zero of $\Psi$ in $\operatorname{Re}(s)<-\rho_{0}$. Thus $\operatorname{Re}\left(p_{k}\right) \geq-\rho_{0}$. Since $\Psi$ has only a finite number of poles in $\operatorname{Re}(s)$ $>0$, all of these being on the line segment $0<\operatorname{Re}(s) \leq \rho_{0}$, we see that only a finite number, say $q_{1}, \cdots, q_{\ell}$, of the $q$ 's lie in $\operatorname{Re}(s)>0$, and they lie on $0<\operatorname{Re}(s) \leq \rho_{0}$. Now (1.23) leads easily to the conclusion that $\Psi^{\prime}(s) \Psi(s)^{-1}$ is invariant under the transformation $s \rightarrow-s$. Since $\Psi^{\prime}(s) \Psi(s)^{-1}=$ $P^{\prime}(s) P(s)^{-1}-Q^{\prime}(s) Q(s)^{-1}$, we conclude from this that if $q_{k}$ is a zero of order $b_{k}$ of $Q$, then $-q_{k}$ is a zero of order $b_{k}$ of $P$, and conversely. It follows that we can label the $p_{k}$ 's in such a way that $p_{k}=-q_{k}$, and then $a_{k}=b_{k}$. This said, consider the function $P(s) / Q(-s)$. It is seen to be an entire function with no zeroes, hence it is of the form $\exp R(s)$, with $R(s)$ entire. Thus $P(s)=Q(-s) \exp R(s)$, and hence $\Psi(s)=Q(-s) \exp R(s) Q(s)^{-1}$; 
moreover, $R(s)=-R(-s)$. Note that the zeroes of $\Psi$ are at $\left\{-q_{k}\right\}_{k \geq 1}$ and the poles of $\Psi$ are at $\left\{q_{k}\right\}_{k \geq 1}$, of orders $\left\{b_{k}\right\}_{k \geq 1}$. It follows that the poles of $\Psi^{\prime}(s) \Psi(s)^{-1}$ are all simple, and are at $\left\{ \pm q_{k}\right\}_{k \geq 1}$. The residue at $q_{k}$ is $-b_{k}$ and the residue at $-q_{k}$ is $b_{k}$. Note that $b_{k} \geq 0$ and is integral. Finally, since $Q$ is entire and of finite order, we know that for some integer $N>0$, we have $\sum_{k \geq 1} b_{k}\left|q_{k}\right|^{-N}<\infty$.

Proposition 3.10. We have

$$
\begin{aligned}
A_{7}(s) & =-\frac{1}{4 \pi} \int_{-\infty}^{\infty} F_{s}^{*}(r) \operatorname{trace}\left(M^{\prime}(i r) M(i r)^{-1}\right) d r \\
& =\sum_{k \geq \ell+1} b_{k} \frac{H\left(i\left(s-\rho_{0}-q_{k}\right)\right)}{s-\rho_{0}-q_{k}}
\end{aligned}
$$

for $\operatorname{Re}(s)>2 \rho_{0}$. The series on the right converges absolutely, uniformly on compact subsets of $C$ disjoint from $\left\{\rho_{0}+q_{k} \mid k \geq \ell+1\right\}$ and defines $a$ meromorphic continuation of the left side to all of $C$. The poles of $A_{7}(s)$, thus continued, are all simple, and are at the points $\left\{\rho_{0}+q_{k} \mid k \geq \ell+1\right\}$. The residue at the pole $\rho_{0}+q_{k}$, is $b_{k} H(0)$.

Proof. We have

$$
\begin{aligned}
A_{7}(s) & =-\frac{1}{4 \pi} \int_{-\infty}^{\infty} F_{s}^{*}(r) \Psi^{\prime}(i r) \Psi(i r)^{-1} d r \\
& =-\frac{1}{4 \pi} \int_{-\infty}^{\infty}\left(\frac{H\left(i\left(s-\rho_{0}-i r\right)\right)}{s-\rho_{0}-i r}+\frac{H\left(i\left(s-\rho_{0}+i r\right)\right)}{s-\rho_{0}+i r}\right) \frac{\Psi^{\prime}(i r)}{\Psi(i r)} d r .
\end{aligned}
$$

Using the fact that $\Psi^{\prime}(i r) / \Psi(i r)$ is invariant under $r \rightarrow-r$, we get

$$
A_{7}(s)=-\frac{1}{2 \pi} \int_{-\infty}^{\infty} \frac{H\left(i\left(s-\rho_{0}-i r\right)\right)}{s-\rho_{0}-i r} \cdot \Psi^{\prime}(i r) \Psi(i r)^{-1} d r
$$

We are now going to shift the contour of integration into the complex half plane $\operatorname{Im}(r) \geq 0$. Let $R>0$, and consider the semicircle of radius $R$ in the upper half $r$-plane, centered at 0 . If we write $\theta(r)=\Psi^{\prime}(i r) \Psi(i r)^{-1}$ for brevity, we see, bearing in mind our discussion above, that $\theta$ has simple poles at $-i q_{k}, k \geq 1$, with residues $i b_{k}, k \geq 1$. Moreover, we also see that of these poles, only the poles at $-i q_{k}, k \geq \ell+1$, lie in the upper half plane. Applying Cauchy's residue theorem to the semicircular contour mentioned above, we find 


$$
\begin{aligned}
& -\frac{1}{2 \pi} \int_{-R}^{R} \frac{H\left(i\left(s-\rho_{0}-i r\right)\right)}{s-\rho_{0}-i r} \theta(r) d r \\
& \quad=\sum_{R} b_{k} \cdot \frac{H\left(i\left(s-\rho_{0}-q_{k}\right)\right)}{s-\rho_{0}-q_{k}}+\frac{1}{2 \pi} \int_{C_{R}} \frac{H\left(i\left(s-\rho_{0}-i z\right)\right)}{s-\rho_{0}-i z} \theta(z) d z,
\end{aligned}
$$

the sum being over those poles that lie within the contour, and where $C_{R}$ is the curved boundary of the contour. Now recall that $\theta(z)=\Psi^{\prime}(i z) \Psi(i z)^{-1}$ and $\Psi$ is the quotient of two entire functions of finite order. By a classical theorem, see e.g. [18, p. 74], there exists a sequence of positive real numbers $\varepsilon_{k}$ approaching zero, and constants $K, m$, such that if $S$ is the union of the closed disks of radius $\varepsilon_{k}$ centred at $q_{k}$ and $-q_{k}, k \geq 1$, then for $z$ in the complement of $S$, we have

$$
|\theta(z)| \leq K|z|^{m}
$$

It follows from this result that we can find a sequence of positive real numbers $R_{j} \rightarrow \infty$ such that on the circles $|z|=R_{j}$, we have

$$
|\theta(z)| \leq K|z|^{m}
$$

We now pick $R$ to be equal to one of these $R_{j}$, and want to estimate the second term on the right side of (3.19) for $R=R_{j}$. Since $\operatorname{Re}(s)>2 \rho_{0}$, and $\operatorname{Im}(z) \geq 0$ for $z$ on $C_{R_{j}}$, we see that $\operatorname{Im}\left(i\left(s-\rho_{0}-i z\right)\right) \geq 0$, so that the estimates of Lemma 3.2 are available, and for any integer $n$, we have $C_{n}>0$ such that $\left|H\left(i\left(s-\rho_{0}-i z\right)\right)\right| \leq C_{n}\left|s-\rho_{0}-i z\right|^{-n}$. In addition $\left|s-\rho_{0}-i z\right| \geq$ $|z|-\left|s-\rho_{0}\right|=R_{j}-\left|s-\rho_{0}\right|$ for $z$ on $C_{R_{j}}$. So using all of this we obtain the estimate

$$
\left|\frac{1}{2 \pi} \int_{C_{R j}} \frac{H\left(i\left(s-\rho_{0}-i z\right)\right)}{s-\rho_{0}-i z} \theta(z) d z\right| \leq K^{\prime} \cdot C_{n} \cdot \frac{\left|R_{j}\right|^{m+1}}{\left(\left|R_{j}\right|-\left|s-\rho_{0}\right|\right)^{n}}
$$

where $K^{\prime}$ is some constant. Since $n$ is at our disposal, we conclude that this integral goes to zero as $R_{j} \rightarrow \infty$. It thus follows from (3.19) that

$$
-\frac{1}{2 \pi} \int_{-\infty}^{\infty} \frac{H\left(i\left(s-\rho_{0}-i r\right)\right)}{s-\rho_{0}-i r} \theta(r) d r=\sum_{k \geq \ell+1} b_{k} \cdot \frac{H\left(i\left(s-\rho_{0}-q_{k}\right)\right)}{s-\rho_{0}-q_{k}}
$$

The sum is over $k \geq \ell+1$, because these are precisely the indices for which the poles of $\theta(r)$ are in the upper half $r$-plane, as we noted above. This proves the first assertion of the proposition. The other assertions follow as in the propositions above, bearing in mind the fact that $\sum b_{k}\left|q_{k}\right|^{-N}<\infty$ for a suitable $N$. 
The net result of all these propositions is seen to be that $z_{\Gamma}$ has a meromorphic continuation to all of $C$, with simple poles. The residues at these various poles are set out above.

We now choose the value of the constant $c=g\left(\varepsilon_{0}\right)$, which was left unspecified in the definition of the auxiliary function $g$ at the beginning of this section. Note that $g\left(\varepsilon_{0}\right)=H(0)$.

By using the results of [7], it can be shown, exactly as in [5], that in our normalization of measures, the volume $\operatorname{vol}(G / \Gamma)$ is a rational number. In fact the manifold $K \backslash G / \Gamma$ has the homotopy type of a compact manifold $M$, and $\operatorname{vol}(G / \Gamma)$ is a rational multiple of the Euler-Poincaré characteristic $E$ of $M$. Moreover, as observed in [5], the numbers $\mathrm{id}_{k}$, $k \geq 1$, are all rational, with denominator depending only on $(G, K)$ and not on $k$. Thus there exists an integer $\kappa>0$ such that $i \operatorname{vol}(G / \Gamma) d_{k}=$ $e_{k} E / \kappa$, where $e_{k}$ is an integer, and $e_{k} E$ and $\mathrm{id}_{k}$ are of like sign. We now choose $H(0)$ to be equal to $4 \kappa$. With this choice, we observe that $z_{\Gamma}$ is meromorphic, with simple poles and integer residues, so that there exists a meromorphic function $Z_{\Gamma}$ (unique up to a multiplicative constant) such that $z_{\Gamma}=Z_{\Gamma}^{\prime} \mid Z_{\Gamma}$. At any pole of $z_{r}$ where the residue is a negative integer equal to $-m$ say, the function $Z_{r}$ has a pole of order $m$, and $Z_{r}$ has a zero of order $m$ wherever $z_{\Gamma}$ has pole with positive residue equal to $m$. For the reader's convenience let us summarize the information about the poles of $z_{\Gamma}$ :

\section{TABLE I}

$$
\begin{gathered}
\text { Pole } \\
s_{j}^{+}=\rho_{0}+i \nu_{j}\left(H_{0}\right) \\
s_{j}^{-}=\rho_{0}-i \nu_{j}\left(H_{0}\right) \\
\rho_{0} \\
\rho_{0}-k \\
0 \\
2 \rho_{0} \\
\rho_{0}+i r_{k} \\
\rho_{0}+q_{k}
\end{gathered}
$$

Residue

$$
\begin{aligned}
& 4 \kappa n_{j} \\
& 4 \kappa n_{j} \\
& \left\{\begin{array}{l}
8 \kappa n_{j}-2 \kappa(d-\operatorname{trace}(M(0))) \\
-2 \kappa(d-\operatorname{trace}(M(0)))
\end{array}\right. \\
& -4 \kappa d \\
& \left\{\begin{array}{l}
4 \kappa-4 e_{0} E \\
4 \kappa-4 e_{0} E-4 \kappa d
\end{array}\right. \\
& 4 \kappa \\
& -4 e_{k} E \\
& 4 \kappa b_{k}
\end{aligned}
$$

$$
\begin{aligned}
& j \geq 1, \nu_{j} \neq 0 \\
& j \geq 1, \nu_{j} \neq 0
\end{aligned}
$$

if for some $j, \nu_{j}=0$

if for no $j, \nu_{j}=0$

$$
k \geq 1, k \neq \rho_{0}
$$

if $\rho_{0}$ is not integral

if $\rho_{0}$ is integral

$$
\begin{gathered}
k \geq 1 \\
k \geq \ell+1
\end{gathered}
$$

Recall here that $d$ is the number of cusps of $\Gamma$. The reason for the dichotomies for the residues at $\rho_{0}$ and 0 is pretty clear: different terms $A_{i}(s)$ contribute to the residues there. Thus, if for some $j, \nu_{j}=0$ (so that 
$0 \in \Lambda$ occurs in the spectrum), the poles $s_{j}^{+}$and $s_{j}^{-}$coalesce and produce the residue $8 \kappa n_{j}$ for that $j$. In this case $s_{j}^{+}=s_{j}^{-}=\rho_{0}$. Moreover, the term $A_{3}(s)$ contributes the residue $-2 \kappa(d-\operatorname{trace}(M(0)))$, in any case. Note that the residue at $\rho_{0}$ is a non-negative even integer. It was to make this integer even that we chose $H(0)=4 \kappa$. As for the residue at 0 , the term $A_{1}(s)$ contributes $4 \kappa$, while $-4 e_{0} E$ comes from $A_{2}(s)$. If $\rho_{0}$ is an integer, the term $A_{4}(s)$ contributes a residue equal to $-4 \kappa d$ at 0 . The residue at $2 \rho_{0}$ comes from the fact that $s_{0}^{-}=2 \rho_{0}$, so that the term $A_{1}(s)$ contributes the residue $4 \kappa$ there.

There are a few possible overlaps in the sets of poles displayed above. It can be checked quite easily that the sets of poles on the first seven lines in the above table are mutually disjoint. As for the set $\left\{\rho_{0}+q_{k} \mid k\right.$ $\geq \ell+1\}$, we shall see that there is no overlap between this set and any of the following sets: $\left\{\rho_{0}+i r_{k} \mid k \geq 1\right\},\left\{\rho_{0}\right\},\left\{2 \rho_{0}\right\},\left\{s_{j}^{-} \mid j \geq 1, \nu_{j} \neq 0\right\}$. Indeed if $\rho_{0}+q_{k}$ belongs to $\left\{\rho_{0}+i r_{k} \mid k \geq 1\right\}$ for some $k$, we would have $q_{k}$ purely imaginary, which is an impossibility because $\Psi$ has no poles on the imaginary axis. For the same reason, $\rho_{0}+q_{k}$ cannot equal $\rho_{0}$. Next, if $\rho_{0}+q_{k}=2 \rho_{0}$ for some $k$, we would have $q_{k}=\rho_{0}$, but since $k \geq \ell+1$ we already know that $\operatorname{Re} q_{k}<0$. Thus $\rho_{0}+q_{k} \neq 2 \rho_{0}$ for any $k$. Finally, if $\rho_{0}+q_{k}=s_{j}^{-}$for some $j$, we would have $q_{k}=i_{j}\left(H_{0}\right)$ for some $j$. This is impossible if $\nu_{j}\left(H_{0}\right)$ is real, because $q_{k}$ cannot be purely imaginary. On the other hand, if $\nu_{j}\left(H_{0}\right)$ is not real, we know that it is purely imaginary and $s_{j}^{-}$lies in $\left(\rho_{0}, 2 \rho_{0}\right]$. It follows that $q_{k}$ would have to lie in $\left(0, \rho_{0}\right]$ which is impossible since $k \geq \ell+1$. Proceeding further in this fashion we see that there are some possible overlaps between the set $\left\{\rho_{0}+q_{k} \mid k \geq \ell+1\right\}$ and the sets $\{0\},\left\{\rho_{0}-k \mid k \geq 1\right\}$, and $\left\{s_{j}^{+} \mid j \geq 1, \nu_{j}\left(H_{0}\right)\right.$ purely imaginary $\}$. We cannot be precise about these overlaps because the location of the poles $q_{k}$ is essentially unknown, as also is the presence of the complementary series $\left\{s_{j}^{+} \mid j \geq 1, \nu_{j}\left(H_{0}\right)\right.$ purely imaginary\}. Of course, when such overlaps occur the residues are to be added up at those locations.

Now denote by $m_{0}$ the integer that equals the residue of $z_{\Gamma}$ at the pole $s=\rho_{0}$. Note that $m_{0}$ is even, and $\geq 0$. We now normalize $Z_{\Gamma}$ by requiring that $\lim _{s \rightarrow \rho_{0}}\left(s-\rho_{0}\right)^{-m_{0}} Z_{\Gamma}(s)=1$. This fixes $Z_{\Gamma}$ completely. We call $Z_{\Gamma}$ the Selberg zeta function of $(G, K, \Gamma)$.

\section{§4. Properties of $Z_{\Gamma}$}

The following properties of $Z_{\Gamma}$ are immediate from Table I of $\S 3$. 
(4.1) $Z_{\Gamma}$ is a meromorphic function, having no poles in $\operatorname{Re}(s)>2 \rho_{0}$.

(4.2) $Z_{T}$ has zeroes at $s_{j}^{+}, s_{j}^{-}, j \geq 1, \nu_{j} \neq 0$. These we call spectral zeroes, as in [5]. These zeroes determine the location of the $\nu_{j}$ which correspond to the spherical representations $U_{j}$ occurring in $L_{d}^{2}(G / \Gamma)$. The order of the zero at $s_{j}^{+}$or $s_{j}^{-}$is $4 \kappa n_{j}$ where $n_{j}$ is the multiplicity of $U_{j}$ in $L_{d}^{2}(G / \Gamma)$. There is also a spectral zero of order $4 \kappa$ at $2 \rho_{0}$. (However, see (4.7) below).

(4.3) There are also certain topological zeroes or poles of $Z_{\Gamma}$. They only occur when $\operatorname{dim}(G / K)$ is even. They occur at $\left\{\rho_{0}+i r_{k} \mid k \geq 1\right\}$ where $\left\{r_{k} \mid k \geq 1\right\}$ are the poles of $r \rightarrow c(r)^{-1} c(-r)^{-1}$ in the upper half plane $\operatorname{Im}(r) \geq 0$. The numbers $\left\{\rho_{0}+i r_{k} \mid k \geq 1\right\}$ are all negative integers, and for $G$ given, they are either all poles or all zeroes of $Z_{\Gamma}$. Whether they are poles or zeroes depends on the sign of the numbers $\left\{\operatorname{id}_{k} \mid k \geq 1\right\}$ where $d_{k}$ is the residue of $c(r)^{-1} c(-r)^{-1}$ at $r_{k}$. The numbers $\left\{\operatorname{id}_{k} \mid k \geq 1\right\}$ are all real and nonzero, and have the same sign. If they are positive, then $Z_{r}$ has poles at $\left\{\rho_{0}+i r_{k} \mid k \geq 1\right\}$; otherwise these are zeroes of $Z_{\Gamma}$. The order of the zero or pole at $\rho_{0}+i r_{k}$ is $\left|4 e_{k} E\right|$, and $e_{k}$ is explicitly computable.

(4.4) The point $\rho_{0}$ may be a zero or a pole of $Z_{\Gamma}$. It will be a zero of $Z_{T}$ if there exists an index $j$ for which $\nu_{j}=0$, i.e. if 0 occurs in the spherical spectrum of $L_{d}^{2}(G / \Gamma)$ and if $8 \kappa n_{j}-2 \kappa(d-\operatorname{trace}(M(0)))$ is positive. In that case, the order of the zero at $\rho_{0}$ is $8 \kappa n_{j}-2 \kappa(d-\operatorname{trace}(M(0)))$, where $n_{j}$ is the multiplicity with which the spherical representation corresponding to $\nu_{j}=0$ occurs in $L_{d}^{2}(G / \Gamma)$. If $\nu_{j} \neq 0$ for any $j$, so that 0 does not occur in the spherical spectrum of $L_{d}^{2}(G / \Gamma)$, then $\rho_{0}$ may still be a pole of $Z_{\Gamma}$, if $d-\operatorname{trace}(M(0))>0$. In that event, the order of this pole is $2 \kappa(d-$ $\operatorname{trace}(M(0)))$. In all other cases, $\rho_{0}$ is not a pole or a zero of $Z_{\Gamma}$.

(4.5) The point $s=0$ may be a zero or a pole of $Z_{\Gamma}$. If $\rho_{0}$ is not an integer and $4\left(\kappa-e_{0} E\right)$ is positive (resp. negative), then $s=0$ is a zero (resp. pole) of $Z_{\Gamma}$ of order $\left|4\left(\kappa-e_{0} E\right)\right|$. If $\rho_{0}$ is an integer, then we have a zero (resp. pole) at $s=0$, of order $\left|4\left(\kappa-e_{0} E-\kappa d\right)\right|$ if $4\left(\kappa-e_{0} E-\kappa d\right)$ is positive (resp. negative). In all other cases, 0 is neither a zero nor a pole of $Z_{\Gamma}$.

(4.6) $Z_{\Gamma}$ has trivial poles all of order $4 \kappa d$ at the points $\left\{\rho_{0}-k ; k\right.$ integral, $\left.k \geq 1, k \neq \rho_{0}\right\}$.

(4.7) $Z_{\Gamma}$ has zeroes of order $4 \kappa b_{k}$ at the points $\left\{\rho_{0}+q_{k} \mid k \geq \ell+1\right\}$. Here the points $q_{k}, k \geq \ell+1$, are the poles of the function $\Psi(s)=\operatorname{det} M(s)$, 
which lie in the half plane $\operatorname{Re}(s)<0$, and $b_{k}$ is the order of the pole at $q_{k}$. It is to be understood that, in the event that some of these zeroes overlap with the other zeroes or poles of $Z_{\Gamma}$ described above, then the orders of those other zeroes or poles are to be augmented or diminished as the case may be, according to the usual conventions.

We now turn to an important property of $Z_{T}$, namely its functional equation. We shall first establish this functional equation for the logarithmic derivative $Z_{\Gamma}$ of $Z_{\Gamma}$.

Define

$$
\theta(s)=z_{\Gamma}(s)-4 \kappa d \frac{\Gamma^{\prime}\left(1+s-\rho_{0}\right)}{\Gamma\left(1+s-\rho_{0}\right)}+\sum_{k=1}^{\ell} \frac{4 \kappa b_{k}}{\left(s-\rho_{0}-q_{k}\right)} .
$$

Adding the extra terms on the right has the following rationale: the term in $\Gamma^{\prime} \mid \Gamma$ serves to kill the poles of $z_{\Gamma}$ at $\rho_{0}-k, k \geq 1$. The last term is introduced to supply the missing poles $\rho_{0}+q_{k}, k=1, \cdots, \ell$.

Now put $\Phi(t)=4 \kappa \operatorname{vol}(G / \Gamma)[Z(\Gamma)] c(i t)^{-1} c(-i t)^{-1}$.

LEMma 4.1. The function

$$
\Theta(s)+\Theta\left(2 \rho_{0}-s\right)+\Phi\left(s-\rho_{0}\right)-4 \kappa \frac{\Psi^{\prime}\left(\rho_{0}-s\right)}{\Psi\left(\rho_{0}-s\right)}
$$

is an entire function.

Proof. This is a routine computation, using Table I of $\S 3$, and the information about the poles of $\Gamma^{\prime} \mid \Gamma, \Psi^{\prime} / \Psi$ and $\Phi$.

Proposition 4.2. We have

$$
\Theta(s)+\Theta\left(2 \rho_{0}-s\right)+\Phi\left(s-\rho_{0}\right)-4 \kappa \frac{\Psi^{\prime}\left(\rho_{0}-s\right)}{\Psi\left(\rho_{0}-s\right)}-\kappa_{5}-\kappa_{6} J(s) \equiv 0
$$

where $\kappa_{5}, \kappa_{6}, J$ are as in $\S 1$.

Proof. The proof is patterned after the proof of Proposition 2.8 of [5]. We shall not spell out all the details since no new principle is involved. Due to the extra terms occurring in the definition of $\Theta$, the manipulations are messier.

Let $\beta(s)$ be the function

$$
\Theta(s)+\Theta\left(2 \rho_{0}-s\right)+\Phi\left(s-\rho_{0}\right)-4 \kappa \frac{\Psi^{\prime}\left(\rho_{0}-s\right)}{\Psi\left(\rho_{0}-s\right)}
$$


Introduce the variable $r=i\left(\rho_{0}-s\right)$ and regard all the above functions as functions of $r$. When so viewed, we shall not change the notation and instead will simply write $\beta(r)=\beta\left(\rho_{0}+i r\right), \Theta(r)=\Theta\left(\rho_{0}+i r\right), \Phi(r)=\Phi\left(\rho_{0}+i r\right)$, $\left(\Psi^{\prime} \mid \Psi\right)(r)=\left(\Psi^{\prime} \mid \Psi\right)\left(\rho_{0}+i r\right)$. Fix $\varepsilon>0$, and choose $F^{*}$ as in [5, p. 16]-then we can find a $p<1$ and $f \in \mathscr{C}^{p}(K \backslash G / K)$ such that $\hat{f}(\nu)=F^{*}(r), r=r(\nu)$. This $f$ is admissible for the trace formula; we now proceed exactly as in [5], and integrate $F^{*}(r) \Theta(r)$ around the rectangular contour in the complex plane with corners at $\pm R \pm\left(\rho_{0}+\varepsilon\right) i$, use Cauchy's theorem on it, and then let $R \rightarrow \infty$. The absolute value of $\Theta(r)$ can be estimated by means of Proposition 2.2, the explicit knowledge of the numbers $d_{k}$, and the location of the poles $r_{k}$. The application of this procedure can then be justified as in [5]. Because of the last term in the definition (4.8) of $\Theta$, there will be a finite number of residues coming from the terms involving $\left(s-\rho_{0}-q_{k}\right)^{-1}$, $k \leq \ell$. However, these eventually cancel because of the extra terms involving $\Psi^{\prime} \mid \Psi$ in the trace formula. The final result is that

$$
\int_{-\infty}^{\infty} F^{*}(r) \beta(r) d r=\kappa_{5} \int_{-\infty}^{\infty} F^{*}(r) d r+\kappa_{6} \int_{-\infty}^{\infty} F^{*}(r) J(r) d r
$$

Since $F^{*}(r)$ can be varied over a wide class of functions, it follows that $\beta(r)=\kappa_{5}+\kappa_{6} J(r)$ for $r$ real. However, these functions are entire, so the assertion is proved.

We may now write down the functional equation for $Z_{\Gamma}$. To do this, observe that $\Theta(s)$ is the logarithmic derivative of the function $\Omega$ defined by

$$
\Omega(s)=Z_{\Gamma}(s) \cdot \Gamma\left(1+s-\rho_{0}\right)^{-4 k d} \cdot \prod_{k=1}^{\ell}\left(s-\rho_{0}-q_{k}\right)^{4 k b_{k}} .
$$

LEMMA 4.3. The following functional equation holds for $\Omega$ :

$$
\begin{aligned}
\Omega\left(2 \rho_{0}-s\right)= & \Omega(s) \Psi\left(\rho_{0}-s\right)^{4 k} \\
& \times \exp \left[\int_{0}^{s-\rho_{0}} \Phi(t) d t+\kappa_{6} \int_{\rho_{0}}^{s} J(t) d t+\kappa_{5}\left(s-\rho_{0}\right)\right]
\end{aligned}
$$

where $\Phi(t)=4 \kappa \operatorname{vol}(G / \Gamma)[Z(\Gamma)] c(i t)^{-1} c(-i t)^{-1}$, and $\kappa_{5}, \kappa_{6}$ and $J$ are as in $\S 1$.

Proof. Proposition 4.2 leads to

$$
\begin{aligned}
\Omega\left(2 \rho_{0}-s\right)= & C \Omega(s) \Psi\left(\rho_{0}-s\right)^{4 \kappa} \\
& \times \exp \left[\int_{0}^{s-\rho_{0}} \Phi(t) d t+\kappa_{6} \int_{\rho_{0}}^{s} J(t) d t+\kappa_{5}\left(s-\rho_{0}\right)\right]
\end{aligned}
$$

where $C$ is some nonzero constant. We claim that $C=1$. We proceed 
exactly as in [5, Theorem 2.9]. Let $m_{0}$ be the order of the zero of $Z_{\Gamma}$ at $s=\rho_{0}$. Then $m_{0}$ is even and $m_{0} \geq 0$. Thus we conclude, as in [5], that $\left(s-\rho_{0}\right)^{-m_{0}} Z_{\Gamma}(s)$ and $\left(\rho_{0}-s\right)^{-m_{0}} Z_{\Gamma}\left(2 \rho_{0}-s\right)$ both approach 1 as $s \rightarrow \rho_{0}$. Multiplying (4.12) by $\left(s-\rho_{0}\right)^{m_{0}}$ and letting $s \rightarrow \rho_{0}$, we conclude that $C=1$. Bear in mind that $|\Psi(0)|=|\operatorname{det} M(0)|$ and none of the $q_{k}$ are zero.

As an immediate consequence, we have

THEOREM 4.4. The following functional equation holds for $Z_{\Gamma}$ :

$$
\begin{aligned}
Z_{\Gamma}\left(2 \rho_{0}-s\right)= & Z_{\Gamma}(s) \cdot\left(\frac{\Gamma\left(1-s+\rho_{0}\right)}{\Gamma\left(1+s-\rho_{0}\right)}\right)^{4 \kappa d} \cdot\left[\Psi\left(\rho_{0}-s\right)\right]^{4 k} \cdot \prod_{k=1}^{\ell}\left(\frac{s-\rho_{0}-q_{k}}{\rho_{0}-s-q_{k}}\right)^{4 k b_{k}} \\
& \times \exp \left[\int_{0}^{s-\rho_{0}} \Phi(t) d t+\kappa_{6} \int_{\rho_{0}}^{s} J(t) d t+\kappa_{5}\left(s-\rho_{0}\right)\right]
\end{aligned}
$$

where $\Phi(t)=4 \kappa[Z(\Gamma)] \operatorname{vol}(G / \Gamma) c(i t)^{-1} c(-i t)^{-1}, \kappa_{5}, \kappa_{6}, J$ are as in $\S 1, d$ is the number of cusps, $\Psi$ is the determinant of the intertwining operator $M$, and $\left\{q_{k} \mid 1 \leq k \leq \ell\right\}$ are the finitely many poles of $\Psi$ in the interval $\left(0, \rho_{0}\right] . \quad b_{k}$ is the order of the pole at $q_{k}$, and $\kappa$ is the integer depending only on the structure of $(G, K)$ which was defined above.

The functional equation (4.13) can be explicated for special choices of $(G, K, \Gamma)$, i.e. whenever the number of cusps, and the function $\Psi$ can be calculated explicitly. In his classic paper, Selberg makes his usual cryptic comments about some such functional equation in the case of $G$ $=S L(2, R)$, cf. [15, p. 78]. However, no further details have appeared anywhere, even in manuscript form, so far as we know, even for that case.

It is to be noted that the term involving $\kappa_{6}$ and $J$ arises only in the cases $G=S U(n, 1), G=S p(n, 1)$ or $G=F_{4}$. In particular, it does not arise in the case $G=S O(n, 1)$, and thus there is no reference to it, even obliquely, in Selberg's paper.

We want to end this section with two miscellaneous properties of $Z_{\Gamma}$; the proofs of these are analogous to the proofs of similar facts in [5], and we shall omit them.

Let $h_{t}, t>0$, be the spherical fundamental solution of the heat equation $\Omega u=c_{0}^{2}(\partial u / \partial t)$, where $c_{0}^{2}=\left|H_{0}\right|^{2}=2 p+8 q$, and $\Omega$ is the Casimir operator of $G$. Then we have $\hat{h}_{t}(\nu)=\exp -\left(r(v)^{2}+\rho_{0}^{2}\right) t$ and $h_{t}$ is admissible for the trace formula. Using it as input, the trace formula reads 


$$
\begin{aligned}
\sum_{j \geq 0} n_{j} \exp -\left(r\left(\nu_{j}\right)^{2}+\rho_{0}^{2}\right) t \\
=[Z(\Gamma)] \operatorname{vol}(G / \Gamma) h_{t}(1) \\
\quad+(4 \pi t)^{-1 / 2} \sum_{r \in C}\left|u_{r}\right| j(\gamma)^{-1} C(h(\gamma)) \exp -\left(\rho_{0}^{2} t+u_{r}^{2} / 4 t\right) \\
\quad+\frac{1}{4 \pi} \int_{-\infty}^{\infty} \exp -\left(r^{2}+\rho_{0}^{2}\right) t \cdot \Psi^{\prime}(i r) \Psi(i r)^{-1} d r \\
+\frac{1}{4}(d-\operatorname{trace}(M(0))) \exp -\rho_{0}^{2} t \\
\quad-\frac{d}{2 \pi} \int_{-\infty}^{\infty} \exp -\left(r^{2}+\rho_{0}^{2}\right) t \cdot \Gamma^{\prime}(1+i r) / \Gamma(1+i r) d r \\
\quad+\kappa_{5} \int_{-\infty}^{\infty} \exp -\left(r^{2}+\rho_{0}^{2}\right) t d r+\kappa_{6} \int_{-\infty}^{\infty} \exp -\left(r^{2}+\rho_{0}^{2}\right) t \cdot J(r) d r .
\end{aligned}
$$

Now define a theta function $\theta_{\Gamma}(t)$ to be equal to the second term on the right side of this formula. Observe that this is the term coming from just the hyperbolic elements in $C_{\Gamma}$. The first term on the right represents the contribution of the central elements, while the other terms on the right arise from the parabolic elements. Proceeding then as in [5], we see that we have the relation

$$
\int_{0}^{\infty} \exp -s\left(s-2 \rho_{0}\right) t \cdot \theta_{\Gamma}(t) d t=8^{-1} \kappa^{-1}\left(s-\rho_{0}\right)^{-1} z_{\Gamma}(s)
$$

so that

$$
\frac{d}{d s}\left(\log Z_{\Gamma}(s)\right)=8 \kappa\left(s-\rho_{0}\right) \int_{0}^{\infty} \theta_{\Gamma}(t) \exp -s\left(s-2 \rho_{0}\right) t d t
$$

This relation is one of the two miscellaneous properties promised above.

Finally, proceeding exactly as in $[5$, p. 31], we obtain the following infinite product for $Z_{\Gamma}$ :

$$
Z_{\Gamma}(s)=C \prod_{\delta \in \operatorname{Prim}} \prod_{\lambda \in L}\left[\left(1-\xi_{\lambda}(h(\delta))\right)^{-1} \exp -s u_{\delta}\right]^{4 \kappa m_{\lambda}} .
$$

Here $C$ is some constant determined by our normalization of $Z_{\Gamma}$, Prim ${ }_{\Gamma}$ is the set of primitive hyperbolic conjugacy classes, $L$ is the semi-lattice of linear forms on $a$ of the form $\sum_{i=1}^{t} m_{i} \alpha_{i}, \alpha_{1}, \cdots, \alpha_{t}$ being the elements of $P_{+}$and $m_{1}, \cdots, m_{t}$ being non-negative integers, $m_{\lambda}$ is the number of distinct $t$-tuples $\left(m_{1}, \cdots, m_{t}\right)$ of non-negative integers such that $\lambda=$ $\sum_{i=1}^{t} m_{i} \alpha_{i}$, and $\xi_{i}$ is the character of $\mathfrak{a}$ corresponding to $\lambda$.

This is the second miscellaneous result which we promised above. 
A number of fragmentary results concerning the non-tempered spherical spectrum can be obtained in the spirit of $[5, \mathrm{p} .23]$. However, to exploit the perturbation method of [14] successfully, we need a trace formula for the discrete part of the decomposition of the representation of $G$ induced from an arbitrary finite dimensional unitary representation of $\Gamma$. Since we do not yet have such a formula, we shall leave matters here for the present.

\section{$\S 5$. Conjugacy classes in $\Gamma$}

The analytic continuation of $Z_{\Gamma}$ which was established in $\S 3$ above has an interesting application to the study of the distribution of conjugacy classes in $\Gamma$. In turn, this is related to certain geometric information about the space $K \backslash G / \Gamma$. We want to describe this application in this section. To avoid awkward formulations, we shall assume that $Z(\Gamma)=\{1\}$. This is no essential loss of generality. Of course, the other assumptions on $\Gamma$ still continue in force.

Let $\gamma \in C_{\Gamma}-\{1\}$. Then the number $u_{r}$ (defined in $\left.\S 1\right)=\beta\left(\log h_{p}(\gamma)\right)$ depends only on the conjugacy class of $\gamma$. Thus, the distribution of conjugacy classes can be studied by studying the numbers $u_{\gamma}$.

The numbers $u_{r}$ also have a geometric interpretation. Remember that $K \backslash G / \Gamma$ has the homotopy type of a compact manifold, and that $\Gamma$ is isomorphic to its fundamental group. We may, therefore, interpret each conjugacy class in $\Gamma$ as a homotopy class of free closed paths on $K \backslash G / \Gamma$. Each such free homotopy class contains a geodesic which is of minimal length among all paths in that class. The number $u_{r}$ is essentially (i.e. up to a fixed constant multiple independent of $\gamma$, and depending only on our choice of invariant metric) the length of the minimal geodesic in the homotopy class corresponding to $\gamma$ (cf. [4]). The numbers $\left\{u_{r} \mid \gamma \in C_{\Gamma}-\{1\}\right\}$ cannot have a finite point of accumulation in $[0, \infty)$, as is evident from the trace formula (cf. (1.47)). The distinct members of the set $\left\{u_{r} \mid \gamma \in C_{\Gamma}\right.$ $-\{1\}\}$ comprise what has been called the length spectrum of $K \backslash G / \Gamma$. Suppose these are $\ell_{1}<\ell_{2}<\ell_{3} \cdots$, and let $m_{i}$ be the number of elements $\gamma$ in $C_{\Gamma}-\{1\}$ for which $u_{r}=\ell_{i}$ - then $m_{i}$ is called the multiplicity of the length $\ell_{i}$, and the set $\left\{\ell_{i}, m_{i}\right\}_{i \geq 1}$ is called the length spectrum with multiplicity.

Recall also that a $\gamma \in \Gamma$ is primitive if it cannot be written in the 
form $\gamma=\delta^{j}$ with $\delta \in \Gamma$ and $j>1$. Every $\gamma \in \Gamma$ can be written uniquely in the form $\gamma=\delta^{j(\gamma)}$ with $\delta$ primitive and $j(\gamma) \geq 1$. We denote by $\operatorname{Prim} \Gamma$ a complete set of representatives for the conjugacy classes of primitive hyperbolic elements in $\Gamma$. The set $\left\{u_{\delta} \mid \delta \in \operatorname{Prim} \Gamma\right\}$ can be ordered in a sequence $0<p_{1}<p_{2}<\cdots$ to yield the primitive length spectrum, $\left\{p_{i}\right\}_{i \geq 1}$. Letting $g_{i}$ be the cardinality of $\left\{\delta \in \operatorname{Prim} \Gamma \mid u_{\delta}=p_{i}\right\}$, we get the primitive length spectrum with multiplicity, viz. the set $\left\{p_{i}, g_{i}\right\}_{i \geq 1}$.

Now for any $\ell>0$, define

$$
\begin{aligned}
& Q_{0}(\ell)=\sum_{\left\{i: p_{i \leq \ell\}}\right.} g_{i} \\
& Q_{1}(\ell)=\sum_{\left\{i: \ell \ell_{i} \leq \ell\right\}} m_{i} .
\end{aligned}
$$

Note that $Q_{0}(\ell)$ is just the number of primitive elements $\delta$ in $C_{\Gamma}$, counting multiplicity, such that $u_{\delta} \leq \ell$. Thus $Q_{0}(\ell)$ is the distribution function of the primitive length spectrum with multiplicity. A similar observation holds for $Q_{1}(\ell)$.

We are going to study the asymptotic behavior of $Q_{0}(\ell)$ and $Q_{1}(\ell)$. $Q_{0}(\ell)$ will be studied first. The assertions about $Q_{1}(\ell)$ will then be deduced from those about $Q_{0}(\ell)$.

In order to do this, we introduce the generalized Dirichlet series

$$
E(s)=\sum_{\delta \in \operatorname{Prim} \Gamma} u_{\delta} \exp -s u_{\delta} .
$$

The strategy will be to study the analytic behavior of $E(s)$ using properties of $z_{\Gamma}$ and then use the Tauberian theorem of Wiener-Ikehara, as is customary in such problems.

It will be convenient to introduce

$$
\begin{gathered}
F(s)=\sum_{r \in C_{\Gamma^{-}}\{1\}} u_{r} j(\gamma)^{-1} \exp -s u_{r} \\
G(s)=\sum_{\gamma \in C_{\Gamma^{-}}\{1\}} u_{r} j(\gamma)^{-1} C(h(\gamma)) \exp \left(\rho_{0}-s\right) u_{r} .
\end{gathered}
$$

Note that $G(s)$ is just a multiple of $z_{\Gamma}(s)$. In fact $G(s)=z_{\Gamma}(s) / 4 \kappa$. Thus $G(s)$ converges if $\operatorname{Re}(s)>2 \rho_{0}$.

Lemma 5.1. (i) Both $F(s), E(s)$ are absolutely convergent if $\operatorname{Re}(s)>2 \rho_{0}$. (ii) We have 


$$
F(s)=\sum_{\delta \in \operatorname{Prim} \Gamma} u_{\delta} \exp -s u_{\delta} /\left(1-\exp -s u_{\delta}\right) \quad \text { for } \operatorname{Re}(s)>2 \rho_{0} .
$$

(iii) The function $E(s)-F(s)$ is holomorphic in $\operatorname{Re}(s)>\rho_{0}$.

Proof. As we saw above, $G(s)$ converges absolutely in $\operatorname{Re}(s)>2 \rho_{0}$. Now consider the coefficient $C(h(\gamma)) \exp \rho_{0} u_{r}$. Clearly $\rho_{0} u_{\gamma}=\rho\left(\log h_{\mathfrak{p}}(\gamma)\right)$. It follows that $C(h(\gamma)) \exp \rho_{0} u_{\gamma}=\varepsilon_{R}^{A}(h(\gamma)) \cdot \prod_{\alpha \in P_{+}}\left(1-\xi_{\alpha}(h(\gamma))^{-1}\right)^{-1}$. Endow $C_{\Gamma}-$ $\{1\}$ with the discrete topology, so that $\gamma \rightarrow \infty$ means, as usual, that $\gamma$ eventually stays outside every finite set. Then we have $u_{r} \rightarrow \infty$ as $\gamma \rightarrow \infty$. It is easy to see that $\left|\xi_{\alpha}(h(\gamma))\right|=\exp \alpha\left(h_{\mathrm{p}}(\gamma)\right), \alpha \in P_{+}$. So $\left|\xi_{\alpha}(h(\gamma))\right| \rightarrow \infty$ as $\gamma \rightarrow \infty$. It follows that $C(h(\gamma)) \exp \rho_{0} u_{\gamma} \rightarrow 1$ as $\gamma \rightarrow \infty$. From this, it is easily concluded that the series for $F(s)$ converges absolutely for $\operatorname{Re}(s)>$ $2 \rho_{0}$. Since the series $E(s)$ consists of terms that occur in $F(s)$, (i) follows.

Next, observe that $C_{\Gamma}-\{1\}$ is the union of $\left\{\delta^{j} \mid j \geq 1\right\}$ as $\delta$ ranges over $\operatorname{Prim} \Gamma$, and $u_{\gamma} j(\gamma)^{-1}=u_{\delta}$ if $\gamma=\delta^{j(r)}$. Thus

$$
\begin{aligned}
F(s) & =\sum_{\delta \in \operatorname{Prim} \Gamma} u_{\delta} \sum_{j \geq 1} \exp -s j u_{\delta} \\
& =\sum_{\delta \in \operatorname{Prim} \Gamma} u_{\delta} \exp -s u_{\delta} /\left(1-\exp -s u_{\delta}\right)
\end{aligned}
$$

which proves (ii).

Next consider $E(s)-F(s)$ for $\operatorname{Re}(s)>2 \rho_{0}$. We have

$$
\begin{aligned}
E(s)-F(s) & =\sum_{\delta \in \operatorname{Prim} \Gamma} u_{\delta} \exp -s u_{\delta}\left(1-\left(1-\exp -s u_{\delta}\right)^{-1}\right) \\
& =\sum_{\delta \in \operatorname{Prim} \Gamma} u_{\delta} \exp -2 s u_{\delta} /\left(1-\exp -s u_{\delta}\right) .
\end{aligned}
$$

It is clear that the series on the right converges absolutely for $\operatorname{Re}(s)>\rho_{0}$, by comparison with the series for $E(s)$. This proves (iii).

Lemma 5.2. There exists $\varepsilon>0$ such that $G(s)$ is meromorphic in $\operatorname{Re}(s)>2 \rho_{0}-\varepsilon . \quad$ This statement holds even when $G=S U(2 n, 1) . \quad$ Moreover, the only singularity of $G(s)$ in the half plane $\operatorname{Re}(s)>2 \rho_{0}-\varepsilon$ is a simple pole at $s=2 \rho_{0}$ with residue 1 .

Proof. Consider $z_{\Gamma}(s)$. In $\S 3$, we had expressed $z_{\Gamma}(s)$ as a sum of seven terms $A_{1}(s), \cdots, A_{7}(s)$. A glance at $\S 3$ shows that the terms $A_{2}(s)$, $A_{3}(s), A_{4}(s), A_{5}(s)$ and $A_{7}(s)$ all have holomorphic continuations to a halfplane of the form $\operatorname{Re}(s)>2 \rho_{0}-\varepsilon$ for some $\varepsilon>0$. As for $A_{1}(s)$, we note that it has a meromorphic continuation to the complex plane, and its poles are all simple and are located at the points $s_{j}^{+}, s_{j}^{-}, j \geq 1$. Now, for $j=0$, we have $\nu_{0}=i \rho$, and so $s_{0}^{+}=0, s_{0}^{-}=2 \rho_{0}$. As we noted in $\S 1$, these $s_{0}^{ \pm}$ 
correspond to the occurrence of the trivial representation in $L^{2}(G / \Gamma)$, and the multiplicity $n_{0}$ with which that representation occurs is 1 . Thus $A_{1}(s)$ has a simple pole at $2 \rho_{0}$ with residue $4 \kappa n_{0}=4 \kappa$. Moreover, the other poles of $A_{1}(s)$ lie either on the line segment $\left[0,2 \rho_{0}\right]$ or on the line $\operatorname{Re}(s)$ $=\rho_{0}$. It follows that for some $\varepsilon>0, A_{1}(s)$ is meromorphic in a half-plane of the form $\operatorname{Re}(s)>2 \rho_{0}-\varepsilon$ and the only pole in that half plane is a simple pole at $2 \rho_{0}$.

It remains to discuss the term $A_{6}(s)$. If $G \neq S U(2 n, 1)$, we have seen above that $A_{6}(s)$ is identically zero. It remains to consider the case $G=$ $S U(2 n, 1)$. We will show that in this case, $A_{6}(s)$ is holomorphic in a halfplane $\operatorname{Re}(s) \geq 2 \rho_{0}-\varepsilon$ for some $\varepsilon>0$. By the results of [21, $\left.\S 9\right]$, summarized in $\S 1$ above, we have

$$
A_{6}(s)=\text { Const. } J_{k, k}\left(F_{f_{s}}\right)
$$

where $k$ is defined by $p=4 k-2$. Thus $k=(p+2) / 4$. Observe that $k$ is integral, in fact, $k=n$. Now $J_{k, k}$ is a sum of distributions of the type $J_{\ell, 0}$ and $J_{\ell, 1}$ where $\ell$ ranges over the set of positive integers $1 \leq \ell \leq k$; thus it is enough to prove our assertion for $J_{\ell, 0}\left(F_{f_{s}}\right)$ and $J_{\ell, 1}\left(F_{f_{s}}\right)$ for $\ell \geq 1$. Consider first $J_{\ell, 0}\left(F_{f_{s}}\right)$. We have

$$
J_{\ell, 0}\left(F_{f_{s}}\right)=\int_{0}^{\infty}\left(D^{2 \ell} F_{s}\right)(u) d u
$$

where $F_{s}(u)=F_{f_{s}}(h), u=u(h)$, and $D$ is the operator $(\sinh u)^{-1} d / d u$. By our choice of $f_{s}$, we have $F_{s}(u)=g(u) \exp \left(\rho_{0}-s\right) u$. Thus the integrand is holomorphic in $s$. Now $g$ being zero near $u=0$, the integral is wellbehaved at the lower limit for any $s$. For large $u$, an easy inductive argument shows that $\left|\left(D^{2 \ell} F_{s}\right)(u)\right|$ is $O\left(\exp \left(\rho_{0}-\operatorname{Re}(s)-2 \ell\right) u\right)$. It follows that the integral converges nicely if $\operatorname{Re}(s)>\rho_{0}-2 \ell$ and thus (5.9) is holomorphic in $\operatorname{Re}(s)>\rho_{0}-2 \ell$ which is much more than what we need. Similarly, we have

$$
J_{\ell, 1}\left(F_{f_{s}}\right)=-\int_{0}^{\infty}\left(D^{2 \ell-1} F_{s}\right)(u) \cosh u d u
$$

Arguing as above, we see that as $u \rightarrow \infty$, we have $\left|\left(D^{2 \ell-1} F_{s}\right)(u)\right|=$ $O\left(\exp \left(\rho_{0}-\operatorname{Re}(s)-2 \ell+1\right) u\right)$, so that the integrand is $O\left(\exp \left(\rho_{0}-\operatorname{Re}(\mathrm{s})\right.\right.$ $2 \ell+2) u$ ), and we have convergence for $\operatorname{Re}(\mathrm{s})>\rho_{0}-2 \ell+2$. Since $\ell$ is at least one, this shows that (5.10) is holomorphic in $\operatorname{Re}(s)>\rho_{0}$, which is better than what is necessary. 
The net result of our considerations is that for some $\varepsilon>0, z_{\Gamma}(s)$ is meromorphic in $\operatorname{Re}(s)>2 \rho_{0}-\varepsilon$, and its only pole in that half-plane is a simple pole at $s=2 \rho_{0}$ with residue $4 \kappa$. Since $G(s)=z_{\Gamma}(s) / 4 \kappa$, our lemma is proved.

Lemma 5.3. There exists $\varepsilon>0$ such that the function $F(s)-G(s)$ is holomorphic in $\operatorname{Re}(s)>2 \rho_{0}-\varepsilon$.

Proof. We have, for $\operatorname{Re}(s)>2 \rho_{0}$,

$$
F(s)-G(s)=\sum_{r \in C_{T^{-}}\{1\}} u_{r} j(\gamma)^{-1}\left(1-C(h(\gamma)) \exp \rho_{0} u_{r}\right) \exp -s u_{r} .
$$

Now consider $1-C(h(\gamma)) \exp \rho_{0} u_{r}$. Because $C(h(\gamma)) \exp \rho_{0} u_{r}$ is positive, we have

$$
\begin{aligned}
1-C(h(\gamma)) \exp \rho_{0} u_{\gamma} & =1-\prod_{\alpha \in P_{+}}\left|1-\xi_{\alpha}(h(\gamma))^{-1}\right|^{-1} \\
& =\left(\prod_{\alpha \in P_{+}}\left|1-\xi_{\alpha}(h(\gamma))^{-1}\right|-1\right) / \prod_{\alpha \in P_{+}}\left|1-\xi_{\alpha}(h(\gamma))^{-1}\right| .
\end{aligned}
$$

The term $\prod_{\alpha \in P_{+}}\left(1-\xi_{\alpha}(h(\gamma))^{-1}\right)$ is of the form $1+\sum_{2} \pm \xi_{\lambda}(h(\gamma))^{-1}$ where the sum is finite and $\lambda$ runs over a finite subset of the semi-lattice $L$. It follows that $1-C(h(\gamma)) \exp \rho_{0} u_{r}$ is dominated in absolute value by a finite sum of terms of the form

$$
\left|\xi_{\lambda}(h(\gamma))^{-1}\right| / \prod_{\alpha \in P_{+}}\left|\left(1-\xi_{\alpha}(h(\gamma))^{-1}\right)\right| \quad(\lambda \in L) .
$$

Now fix $\lambda \in L$, and consider the series

$$
P_{\lambda}(s)=\sum_{\gamma \in C_{\Gamma^{-}}\{1\}} u_{\gamma} j(\gamma)^{-1}\left|\xi_{\lambda}(h(\gamma))\right|^{-1} \prod_{\alpha \in P_{+}}\left|\left(1-\xi_{\alpha}(h(\gamma))^{-1}\right)\right| \exp -s u_{\gamma} .
$$

In this series $\left|\xi_{\gamma}(h(\gamma))^{-1}\right|=\exp -\lambda\left(h_{p}(\gamma)\right), \lambda$ being purely imaginary on $h_{t}(\gamma)$. On the other hand, if $\lambda_{0}=\lambda\left(H_{0}\right)$, then we have $\lambda\left(h_{p}(\gamma)\right)=\lambda_{0} u_{r}$. Thus

$$
P_{\lambda}(s)=\sum_{\gamma \in C_{\Gamma^{-}}\{1\}} u_{\gamma} j(\gamma)^{-1} \prod_{\alpha \in P_{+}}\left|\left(1-\xi_{\alpha}(h(\gamma))^{-1}\right)\right|^{-1} \exp -\left(s+\lambda_{0}\right) u_{\gamma}
$$

and, of course, $\lambda_{0}$ is positive, since each $\lambda \in P_{+}$is positive on $H_{0}$. Because $\left|\xi_{\alpha}(h(\gamma))^{-1}\right| \rightarrow 0$ as $\gamma \rightarrow \infty$, we see by comparing this series with the series for $F(s)$ that $P_{2}(s)$ converges absolutely as long as $\operatorname{Re}(s)>2 \rho_{0}-\lambda_{0}$. Now the series $F(s)-G(s)$ is dominated by a sum of finitely many series of the form $P_{\lambda}(s)$ and if we choose $\varepsilon>0$ small enough, we conclude that the series defining $F(s)-G(s)$ converges absolutely in some half-plane $\operatorname{Re}(s)$ $>2 \rho_{0}-\varepsilon$. Since each term is clearly holomorphic, the lemma is proved. 
It follows from Lemmas 5.1, 5.2, 5.3 that $E(s)$ is meromorphic in some half-plane of the form $\operatorname{Re}(s)>2 \rho_{0}-\varepsilon$ with $\varepsilon>0$, and that the only singularity of $E(s)$ in that half-plane is a simple pole at $s=2 \rho_{0}$ with residue 1. The stage is set for an application of the Tauberian theorem of WienerIkehara.

To carry out this application, let us define $A(t)$ for $t>0$ by $A(t)=$

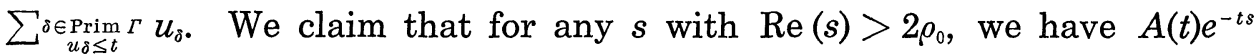
$\rightarrow 0$ as $t \rightarrow \infty$. Indeed, if $\operatorname{Re}(s)>2 \rho_{0}$, then we have $s=s^{\prime}+\varepsilon$ with $\varepsilon>0$ and $\operatorname{Re}\left(s^{\prime}\right)>2 \rho_{0}$ for a suitable $\varepsilon$. Write $A(t) e^{-t s}=\left(A(t) e^{-t s^{\prime}}\right) e^{-t_{\varepsilon}}$. Since

$$
\left|A(t) e^{-t s^{\prime}}\right|=A(t) e^{-t \operatorname{Re}\left(s^{\prime}\right)} \leq \sum_{\substack{\delta \in \operatorname{Prm} \Gamma \\ u_{\delta} \leq t}} u_{\delta} e^{-u_{\delta} \operatorname{Re}\left(s^{\prime}\right)},
$$

and the series on the right converges, $A(t) e^{-t s^{\prime}}$ is bounded, so our claim is proved. It follows from this claim that for $\operatorname{Re}(s)>2 \rho_{0}$, we have

$$
\int_{0}^{\infty} A(t) e^{-t s} d t=\frac{1}{s} \int_{0}^{\infty} e^{-t s} d A(t)
$$

Now the integral on the right is a Stieltjes integral, and the function $A(t)$ has jumps of size $u_{\delta}$ at $t=u_{\delta}$ so

$$
\int_{0}^{\infty} e^{-t s} d A(t)=\sum u_{\delta} e^{-s u_{\delta}}=E(s)
$$

Thus if we write

$$
H(s)=\int_{0}^{\infty} A(t) e^{-t s} d t,
$$

we have that $H(s)=E(s) / s$. In particular, $H(s)$ is meromorphic in a halfplane $\operatorname{Re}(s)>2 \rho_{0}-\varepsilon$ for some $\varepsilon>0$, and has only a simple pole at $s=2 \rho_{0}$ in that half-plane with residue $1 / 2 \rho_{0}$. Now an application of WienerIkehara's theorem yields

$$
2 \rho_{0} A\left(t / 2 \rho_{0}\right) \sim e^{t} \quad \text { as } t \rightarrow \infty
$$

or

$$
\sum_{2 \rho_{0} u_{\delta} \leq t} 2 \rho_{0} u_{\delta} \sim e^{t} \quad \text { as } t \rightarrow \infty .
$$

Now put $v_{\delta}=\exp 2 \rho_{0} u_{\delta}$ and for any $x>0$, put

$$
\omega(x)=\sum_{v_{\delta} \leq x} \log v_{\delta} .
$$

Then (5.18) reads 


$$
\omega(x) \sim x \quad \text { as } x \rightarrow \infty \text {. }
$$

This said, let

$$
\pi(x)=\sum_{\substack{\delta \in \operatorname{Prim} \\ v \delta \leq x}} 1 .
$$

Then it is well known that $\omega(x) \sim x$ is equivalent to

$$
\pi(x) \sim x / \log x \quad \text { as } x \rightarrow \infty \text {. }
$$

Now,

$$
Q_{0}(\ell)=\sum_{\substack{\delta \in \operatorname{Prim} \\ u_{\delta} \leq \ell}} 1=\pi\left(\exp 2 \rho_{0} \ell\right) \sim \exp 2 \rho_{0} \ell / 2 \rho_{0} \ell \quad \text { as } \ell \rightarrow \infty .
$$

As for $Q_{1}(\ell)$, we have

$$
\begin{aligned}
Q_{0}(\ell)= & \sum_{\substack{\delta \in \operatorname{Prim} \\
u_{\delta} \leq \ell}} 1 \leq Q_{1}(\ell)=\sharp\left\{(\delta, j) \mid \delta \in \operatorname{Prim} \Gamma, j \geq 1, j u_{\delta} \leq \ell\right\} \\
& \leq \sum_{\substack{\delta \in \operatorname{Prim} \\
u_{\delta} \leq \ell}} \ell / u_{\delta}=\int_{0}^{\ell} \frac{\ell}{y} d Q_{0}(y)=Q_{0}(\ell)+\int_{0}^{\ell}\left(\ell / y^{2}\right) Q_{0}(y) d y .
\end{aligned}
$$

Since both sides of this inequality are asymptotic to $\exp 2 \rho_{0} \ell / 2 \rho_{0} \ell$, we have $Q_{1}(\ell) \sim \exp 2 \rho_{0} \ell / 2 \rho_{0} \ell$ as $\ell \rightarrow \infty$. Thus we have proved:

Proposition 5.4.

$$
\begin{cases}Q_{0}(\ell) \sim \exp 2 \rho_{0} l / 2 \rho_{0} \ell & \text { as } \ell \rightarrow \infty \\ Q_{1}(\ell) \sim \exp 2 \rho_{0} \ell / 2 \rho_{0} \ell & \text { as } \ell \rightarrow \infty .\end{cases}
$$

\section{Appendix}

Let

$$
g_{t}(x)=\frac{1}{\sqrt{4 \pi t}} \exp \left(-x^{2} / 4 t\right) \quad(x \in R, t>0)
$$

Then the Fourier-Laplace transform of $g_{t}$ is given by

$$
\hat{g}_{t}(z)=e^{-z^{2 t}} \quad(z \in C) .
$$

Our objective here will be to establish the following estimate.

Lemma. Let $\mathscr{S}_{0}$ be a strip in the complex plane parallel and centered with respect to the $x$-axis. Let $k$ and $\ell$ be non-negative integers-then there exists a positive integer $N=N(k, \ell)$ and a positive constant $C=C(k, \ell)$ such that 


$$
\sup _{z_{0} \in \mathscr{s}_{0}}\left|\left(1+z_{0}^{2}\right)^{k} \frac{d^{\ell}}{d z^{\ell}} \hat{g}_{t}\left(z_{0}\right)\right| \leq C \cdot t^{-N} \quad(0<t \leq 1) .
$$

The proof is spelled out in the following lines. We begin with a series of reductions.

The operational calculus for Fourier transforms tells us that

$$
\left(1+z_{0}^{2}\right)^{k} \frac{d^{\ell}}{d z^{\ell}} \hat{g}_{t}\left(z_{0}\right)
$$

is equal to

$$
\left((\sqrt{-1})^{\ell}(1-\Delta)^{k}\left(x^{\ell} g_{t}\right)\right)^{\wedge}\left(z_{0}\right),
$$

$\Delta$ being, of course, $d^{2} / d x^{2}$. Use Leibnitz's rule to write

$$
\left((\sqrt{-1})^{\ell}(1-\Delta)^{k}\left(x^{\ell} g_{t}\right)\right)^{\wedge}\left(z_{0}\right)
$$

as a finite sum of terms of the form

$$
C_{p, q} \cdot\left(x^{p} \frac{d^{q}}{d x^{q}} g_{t}\right)^{\wedge}\left(z_{0}\right)
$$

Then it will be enough to estimate

$$
\left(x^{p} \frac{d^{q}}{d x^{q}} g_{t}\right)^{\wedge}\left(z_{0}\right)
$$

The argument now falls into two parts, according to whether $q$ is even or odd. We shall deal explicitly with the first possibility, the discussion of the second being similar. So suppose that $q$ is even, say $q=2 n$. Since $g_{t}$ is the fundamental solution to the heat equation on the line, one has

$$
\frac{d^{2}}{d x^{2}} g_{t}=\frac{d}{d t} g_{t}
$$

Consequently,

$$
\frac{d^{2 n}}{d x^{2 n}} g_{t}=\frac{d^{n}}{d t^{n}} g_{t}
$$

which implies that we need only estimate

$$
\left(x^{p} \frac{d^{n}}{d t^{n}} g_{t}\right)^{\wedge}\left(z_{0}\right)
$$

For this purpose, note that 


$$
\frac{d^{n}}{d t^{n}} g_{t}=P_{n}(x, t) g_{t}
$$

where $P_{n}$ is a rational function of $x$ and $t$, determined recursively by the scheme

$$
P_{n}=P_{n-1}^{\prime}+P_{1} P_{n-1}
$$

$P_{1}$ being given by

$$
P_{1}(x, t)=\frac{1}{2}\left[\frac{x^{2}}{2 t^{2}}-\frac{1}{t}\right]
$$

It then follows readily that

$$
P_{n}=\sum_{i=0}^{n-1} P_{1}^{i} P_{n-(i+1)}^{\prime}
$$

We can therefore say that there exist an integer $N(n)>n$ and a polynomial $Q_{n}$ in $x$ and $t$ such that

$$
\frac{d^{n}}{d t^{n}} g_{t}=\frac{1}{t^{N(n)}} \cdot\left[Q_{n}(x, t)\right]
$$

Restricting $t$ to the interval ]0,1], we are thus reduced to estimating

$$
\frac{1}{t^{N(r)}} \cdot\left(x^{r} g_{t}\right)^{\wedge}\left(z_{0}\right)
$$

The factor $1 / t^{N(r)}$ will only play a role in the end, so it may be set aside for the moment. At this point, let us recall that $z_{0}$ lies in the strip $\mathscr{S}_{0}$. Thanks to the maximum principle, we can freeze the imaginary part of $z_{0}$ and perform our calculations along a horizontal line. In other words, the following result serves to complete our discussion.

LEMMA. Let $z_{0}=x_{0}+\sqrt{-1} y_{0}$ ( $x_{0}$ variable, $y_{0}$ fixed $)$. Let $r$ be a nonnegative integer-then there exists a positive constant $C=C\left(r, z_{0}\right)$ such that

$$
\left.\left.\sup _{-\infty<x_{0}<+\infty}\left|\left(x^{r} g_{t}\right)^{\wedge}\left(z_{0}\right)\right| \leq C \quad(t \in] 0,1\right]\right) .
$$

Proof. We have

$$
\begin{aligned}
\left|\left(x^{r} g_{t}\right)^{\wedge}\left(z_{0}\right)\right| & =\frac{1}{\sqrt{4 \pi t}}\left|\int_{-\infty}^{\infty} x^{r} e^{-x^{2} / 4 t} e^{\sqrt{-1} z_{0} x} d x\right| \\
& \leq \frac{1}{\sqrt{4 \pi t}} \int_{-\infty}^{\infty}|x|^{r} e^{-x^{2} / 4 t} e^{-y_{0} x} d x
\end{aligned}
$$




$$
\begin{aligned}
& =\frac{1}{\sqrt{4 \pi t}}\left[\int_{0}^{\infty} x^{r} e^{-x^{2} / 4 t} e^{-y_{0} x} d x+\int_{0}^{\infty} x^{r} e^{-x^{2} / 4 t} e^{y_{0} x} d x\right] \\
& =\frac{1}{\sqrt{4 \pi t}}[I(t)+J(t)] \quad \text { (say). }
\end{aligned}
$$

In the integrals defining $I(t)$ and $J(t)$, make the change of variable $x^{2}=$ $4 t u$-then

$$
\left\{\begin{array}{l}
I(t)=2^{r / 2} t^{r / 2+1 / 2} \int_{0}^{\infty} u^{r / 2-1 / 2} e^{-u} e^{-2 y_{0} \sqrt{t} \sqrt{u}} d u \\
J(t)=2^{r / 2} t^{r / 2+1 / 2} \int_{0}^{\infty} u^{r / 2-1 / 2} e^{-u} e^{2 y_{0} \sqrt{t} \sqrt{u}} d u .
\end{array}\right.
$$

As functions of the parameter $t, I$ and $J$ are certainly continuous. Cancelling the $\sqrt{t}$, our assertion follows immediately.

\section{REFERENCES}

[1] De George, D., Length spectrum for compact locally symmetric spaces of strictly negative curvature, Ann. Sci. E.N.S., vol. 10 (1977), pp. 133-152.

[2] Faddeev, L., Expansion in eigenfunctions of the Laplace operator on the fundamental domain of a discrete group on the Lobacevskii plane, AMS Transl. Trudy (1967), pp. 357-386.

[3] Gangolli, R., Asymptotic behavior of spectra of compact quotients of certain symmetric spaces, Acta Math., vol. 121 (1968), pp. 151-192. [See also, T. Eaton (Thesis, University of Washington, 1972).]

[4] - On the length spectra of certain compact manifolds of negative curvature, J. Diff. Geom., vol. 22 (1977), pp. 403-424.

[5] - Zeta functions of Selberg's type for compact space forms of symmetric spaces of rank one, Illinois J. Math., vol. 21 (1977), pp. 1-42.

[ 6 ] Gangolli, R. and Warner, G, On Selberg's trace formula, J. Math. Soc. Japan, vol. 27 (1975), pp. 328-343.

[ 7 ] Harder, G., A Gauss-Bonnet formula for discrete arithmetically defined groups, Ann. Sci. E.N.S., vol. 4 (1971), pp. 409-455.

[8] Harish-Chandra, Spherical functions on a semi-simple Lie Group (I, II), Amer. J. Math., vol. 80 (1958), pp. 241-310, 533-613.

[ 9 ] Hejhal, D., The Selberg trace formula and the Riemann zeta function, Duke Math. J., vol. 43 (1976), pp. 441-482.

[10] Huber, H., Zur Analytische Theorie Hyperbolischer Raumformen und Bewegungsgruppen I, Math. Ann., vol. 138 (1959), pp. 1-26.

[11] Landau, E., Handbuch der Primzahlen, Second Edition, Chelsea, 1953.

[12] Langlands, R., On the functional equations satisfied by Eisenstein series, Springer Lecture Notes, no. 544.

[13] Osborne, S. and Warner, G., Multiplicities of the integrable discrete series: The case of a non-uniform lattice in an $\boldsymbol{R}$-rang one semi-simple group, J. Funct. Analysis, vol. 30 (1978), 287-310.

[14] Randol, B., Small eigenvalues of the Laplace operator on compact Riemann surfaces, Bull. Amer. Math. Soc., vol. 80 (1974), pp. 996-1000. 
[15] Selberg, A., Harmonic analysis and discontinuous groups in weakly symmetric Riemannian spaces with applications to Dirichlet series, J. Indian Math. Soc., vol. 20 (1956), pp. 47-87.

[16] Tanaka, S., Selberg's trace formula and spectrum, Osaka J. Math., vol. 3 (1966), pp. 205-216.

[17] Trombi, P. and Varadarajan, V., Spherical transforms on semi-simple Lie groups, Ann. of Math., vol. 94 (1971), pp. 246-303.

[18] Valiron, G., Fonctions Entieres et Fonctions Meromorphes d'une Variable, Memorial des Sciences Mathematiques, 1925.

[19] Varadarajan, V., Harmonic analysis on real reductive groups, Springer Lecture Notes, no. 576.

[20] Venkov, A., Expansions in automorphic eigenfunctions of the Laplace-Beltrami operator in classical symmetric spaces of rank 1 and the Selberg trace formula, Proc. Steklov Inst. Math., vol. 125 (1973), pp. 6-55.

[21] Warner, G., Selberg's trace formula for non-uniform lattices: The $\boldsymbol{R}$-rank one case, Advances in Math. Studies, vol. 6 (1979), pp. 1-142.

Department of Mathematics

University of Washington

Seattle, Washington 98195 